\title{
INTRODUCING A PERSONAL-ORIGINALITY APPROACH FOR SCORING CHILDREN'S DIVERGENT THINKING
}

A Dissertation
presented to
the Faculty of the Graduate School
at the University of Missouri
In Partial Fulfillment
of the Requirements for the Degree
Doctor of Philosophy
br. Louis Manfra, Dissertation Supervisor
May 2021
James A. Larsen


The undersigned, appointed by the dean of the Graduate School, have examined the dissertation entitled

\section{INTRODUCING A PERSONAL-ORIGINALITY APPROACH FOR SCORING CHILDREN'S DIVERGENT THINKING}

presented by James A. Larsen, a candidate for the degree of doctor of philosophy, and hereby certify that, in their opinion, it is worthy of acceptance.

Professor Louis Manfra

Professor Duane Rudy

Professor Rachel Thibodeau-Nielsen

Professor Kristy vanMarle 


\section{DEDICATION}

This work is dedicated to my grandfather, William Lenhart Groth, Jr., who impressed upon me the importance and power of scientific inquiry. Thank you for modeling what it means to be a lifelong learner and educator. You taught me to view the world as a series of questions, many of which are never truly answered.

"Like poetry in motion, wingtips fly and awed by its beauty, I can but stare. Soaring with the elements eye to eye, a peregrine falcon masters the sky."

-Emile J. Pinet 


\section{ACKNOWLEDGEMENTS}

First and foremost, I would like to express my gratitude to my advisor and mentor, Dr. Louis Manfra. You somehow managed to transform an unfocused undergraduate research assistant into a determined and well-rounded scholar. This document, and the countless hours of hard work it represents, is a testament to the support, patience, and guidance you have provided throughout the years. Thank you for always demanding the very best from me. I will treasure the many lessons I have learned under your supervision and the memories of fine wines, aged spirits, and wonderful adventures we have shared for the rest of my days.

I would also like to thank my esteemed doctoral committee members. Dr. Duane Rudy, you have modeled how to be thoughtful and respectful in all aspects of academic life. I am not sure if I ever told you this, but the conversations we have had over the years are among my fondest memories of graduate school. Dr. Rachel Thibodeau, you have helped me think critically about my work and its implications. I cannot overstate how much I appreciate the many opportunities you have given me to help broaden my knowledge and skillset. Dr. Kristy vanMarle, your words of encouragement and the fresh perspective you brought to our meetings helped me consider new ways of thinking about my research area. Thank you all for helping me produce a dissertation we can be proud of.

Finally, I would like to thank my family for their unwavering support. Mom and dad, you have always instilled in me the importance of diligence and commitment. You helped me recognize that when I put my head down and focus on the challenge in front of me, I am a force to be reckoned with. To my brothers and sister, you have always challenged me to be better. I can attribute much of my success to the invaluable lessons learned from childhood beatdowns and scholarly debates around the dinner table. To my life partner, Katie, you have been with me through all the highest highs and lowest lows that graduate training has to offer. You helped me believe in myself when it was not easy to, and I can confidently say I would not have achieved this milestone without you. And to my son, Landon Burke Larsen, thank you for being an endless source of joy and motivation. I am certain you will surpass your old man in every measurable manner, but I hope we can both look back on this small achievement with pride. 


\section{TABLE OF CONTENTS}

ACKNOWLEDGEMENT .ii

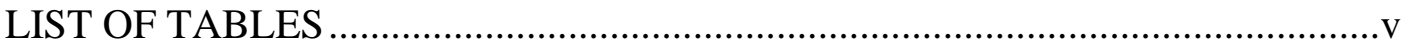

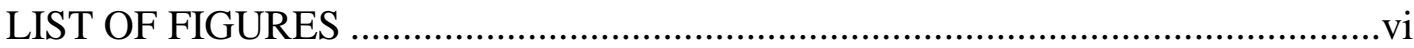

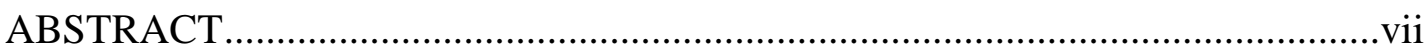

CHAPTER 1: LITERATURE REVIEW …...................................................

Measuring Divergent Thinking ..............................................................2

The Personal-Originality Approach ....................................................

The Role of Time in Divergent Thinking ..................................................

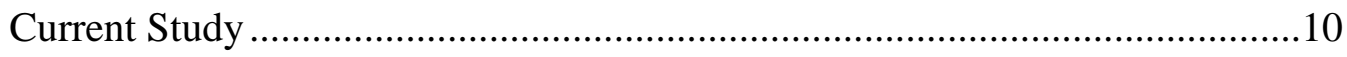

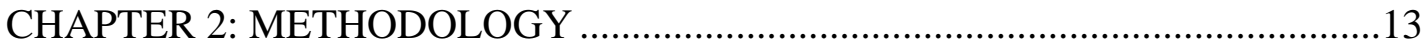

Recruitment and Participants ................................................................ 13

Procedure and Measures ..................................................................... 13

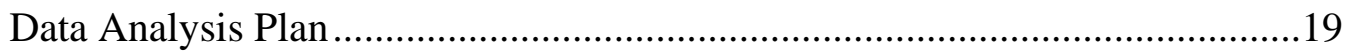

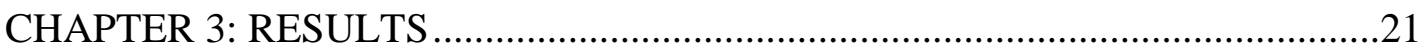

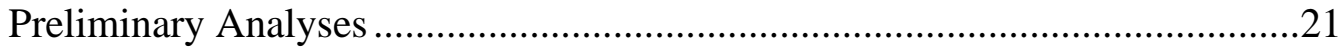

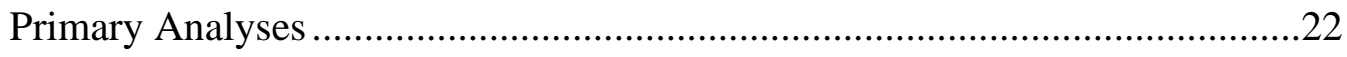

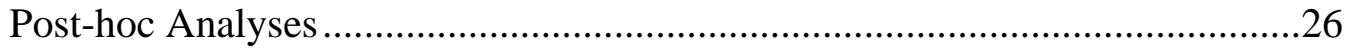

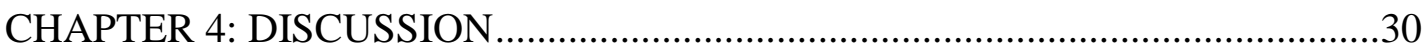

Evidence for Construct Validity ............................................................ 30

The Role of Incubation in Children's Divergent Thinking.............................31

Child Characteristics and Divergent Thinking Scores..................................32

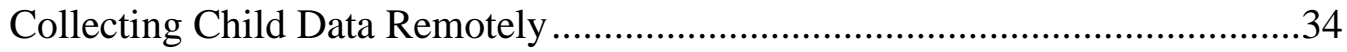


Limitations, Implications, and Future Directions .......................................36

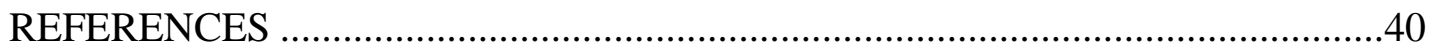

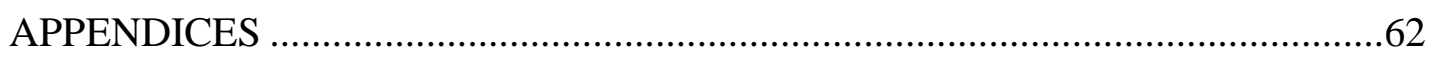

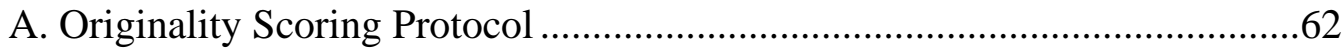

B. Measures and Task Instructions .........................................................64

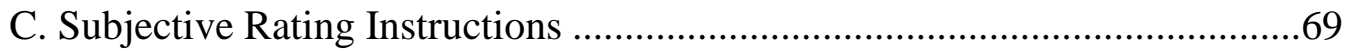

D. Complete Ideational Pools for Divergent Thinking Tasks.........................70

E. Linear Regression Assumptions Tests...................................................76

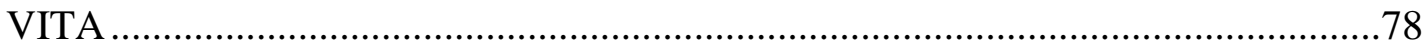




\section{LIST OF TABLES}

Table

1. Descriptive Statistics for All Primary Variables.........................................48

2. Intraclass Correlation Coefficients for Subjective Ratings.........................49

3. Correlations Among IT Scores and Primary Study Variables ......................50

4. Correlations Among AUT Scores and Primary Study Variables...................51

5. Correlations Among Divergent Thinking Task Outcomes ...........................52

6. Results of Linear Regression Analyses Examining the Influence of Child Age and Task-Specific Fluency on Personal Originality...........................53

7. Hierarchical Linear Regression Exploring the Effects of Age and PersonalOriginality Scores on Children's Combined Drawing/Title Scores ..............54 


\section{LIST OF FIGURES}

Figure

1. Sample Drawing with Title for Performance Measure of Creativity..............55

2. Screenshot of Zoom Session Screen-Share During Instances Task...............56

3. Screenshot of Zoom Session Screen-Share During Alternative Uses Task...57

4. Coding Raw Responses for Objective Originality Scoring .........................58

5. Developmental Trajectories of IT Fluency and Originality Scores ...............59

6. Developmental Trajectories of AUT Fluency and Originality Scores..........60

7. Visual Representation of Within-Session Personal Originality Mediating the Relation Between Age and Combined Drawing/Title Creativity Ratings .....61 


\title{
INTRODUCING A PERSONAL-ORIGINALITY APPROACH FOR SCORING CHILDREN'S
}

\section{DIVERGENT THINKING}

James A. Larsen

Dr. Louis Manfra, Dissertation Supervisor

\begin{abstract}
Divergent thinking (DT), which can be described as the ability to conceive of and express a variety of novel solutions to open-ended problems (Gibson et al., 2009), is one of the most commonly studied constructs related to creative thinking (Runco \& Acar, 2012). There is considerable evidence that DT is predictive of later creative behavior and achievement (Benedek et al., 2014; Cramond et al., 2005; Kim, 2008). However, current methods for studying DT rely extensively on cross-sectional comparisons of young children, which may diminish the ability to detect key indicators of DT, such as childlevel originality. The current study introduces a personal-originality (PO) approach for scoring children's performance in common DT tasks. While originality is often assessed in terms of statistical rarity (i.e., objective scoring) or subjective ratings, the PO scoring approach used in the current study captures DT at the child level through the strategic use of incubation periods. That is, children demonstrate $\mathrm{PO}$ by producing new ideas after time away from the task. The current study explored the effects of both short (i.e., 2 minutes) and long (i.e., several days) incubation periods on 42 three- to six-year-olds' DT performance.
\end{abstract}




\section{CHAPTER 1: LITERATURE REVIEW}

Promoting children's creative thinking abilities has become a priority among educators, scholars, policy makers, and government officials in the United States and abroad (Cheung, 2012; McClain \& Pfeiffer, 2012; Nicholson \& Moran, 1986; Pang, 2015; Plucker et al., 2011; Roberts, 2006). In support of this initiative, stakeholders have attempted to identify and harness the skills that underly creative thought and behavior. Several indicators of creative potential have been studied extensively. Some aspects of creative potential are considered relatively stable characteristics of the individual (e.g., personality traits) while others are more malleable (e.g., cognitive abilities). The latter category represents a promising avenue for intervention work and is therefore the central focus of researchers interested in nurturing children's creative potential. Perhaps the most thoroughly investigated indicator of creative potential on the malleable end of the spectrum is divergent thinking (Runco \& Acar, 2012).

Divergent thinking (DT) can be described as the ability to conceive of and generate a variety of novel solutions to open-ended problems (Gibson et al., 2009). Originally coined “divergent production” by J.P. Guilford (1967) in his structure of intellect theory, DT allows individuals to access various domains of background knowledge relevant to a given task (Paletz \& Schunn, 2010) and to mentally transform thoughts and ideas in new and interesting ways (Kilgour, 2006). It is important to note that performance on divergent thinking tests should not be confused with creativity (Runco et al., 2016) because it does not guarantee creative achievement. That is, individuals might think divergently without ever generating a "novel and useful" product 
(Plucker et al., 2004, P. 91). Instead, if properly assessed, divergent thinking can be used for identifying creative potential (Guilford, 1967).

There is considerable evidence that DT is predictive of later creative behavior and achievement (Benedek et al., 2014; Cramond et al., 2005; Kim, 2008; Plucker, 1999). For example, Kyung Hee Kim's (2008) meta-analysis of 44 studies found that DT test scores were better predictors of creative achievement across several domains (e.g., writing, art, science) compared to general intelligence scores. Similarly, Jonathan Plucker (1999) found that divergent thinking scores during childhood were predictive of creative achievement (e.g., awards, inventions, publications) 22 years later after controlling for intelligence scores. However, it is possible that the ways previous researchers have measured divergent thinking provide only partial estimates of children's overall creative potential.

\section{Measuring Divergent Thinking}

Since the introduction of the construct in the 1960s, several researchers have developed and validated tests for measuring DT. These tests typically involve a battery of verbal and/or figural tasks that examine various dimensions of divergent thought. Verbal tasks require participants to consider open-ended problems and produce verbal responses. For example, in Guilford's (1967) alternative uses task, participants are asked to list all the ways one might use a common object (e.g., uses for a brick). Similarly, Wallach and Kogan's (1965) instances task prompts participants to name things that belong to a specific category (e.g., things that make noise). Figural tests, on the other hand, are used to capture creative potential without the high language demands of verbal tasks. For example, in the picture completion task of the Torrance Test of Creative Thinking 
(TTCT; 1966), participants are asked to use incomplete figures to create pictures.

Irrespective of the tools used, researchers measure DT both in terms of the quantity and quality of participants' responses.

\section{Dimensions of Divergent Thinking}

There are four scoring criteria that are frequently used to evaluate DT performance: fluency, flexibility, elaboration, and originality. Fluency is thought of as the productive aspect of DT and is often operationalized as the sheer number of responses an individual generates. Flexibility is considered the capacity to produce different kinds of ideas. It is measured by the number of discrete categories represented across the entire pool of responses. The third component, elaboration, captures the vividness of a response or set of responses. It is evaluated in terms of the amount of detail participants attach to their ideas. The fourth and final dimension common across DT measures is originality, which involves the novelty or uniqueness of an individual's ideas. Conceptually, originality is widely accepted as the characteristic of DT most closely related to raw creativity (Forthmann et al., 2020). However, there is disagreement as to how originality should be operationalized. The following section provides an overview of originality scoring systems frequently used in DT research.

\section{Originality Scoring}

The most common methods for exploring divergent thinking can be separated into two broad categories distinguished by the objective versus subjective approach used in scoring (Silvia, Winterstein, Willse, et al., 2008). Generally speaking, objective originality scoring attempts to capture the rarity of an individual's responses relative to a sample or population, while subjective originality scoring involves the social appraisal 
(i.e., external ratings) of responses.

\section{Objective Scoring Approaches}

In objective scoring, originality is operationalized in terms of the statistical rarity of an individual's responses to an open-ended question. Three of the most common objective scoring approaches are frequency, weighted, and ratio scoring. Frequency scoring operationalizes originality as the number of responses an individual produces that are statistically rare at a specified threshold (e.g., $<10 \%)$ based on the study sample or measurement-specific norming samples. Weighted scoring is an extension of frequency scoring that recognizes different levels of rarity. For example, an idea produced by less than $1 \%$ of the sample is recognized as rarer than an idea produced by $5-10 \%$ of the sample. Though both of these empirically-based approaches are celebrated for their uniformity, they are often criticized because quantity (i.e., fluency) and quality (e.g., originality) subscales are confounded (Hocevar, 1979; Nusbaum \& Silvia, 2011). That is, the greater the number of ideas produced, the higher the likelihood that produced ideas will include original, flexible, and more elaborate responses, resulting in poor discriminant validity of theoretically independent dimensions of DT.

To overcome this limitation, several proponents of objective scoring have turned to ratio scoring. In ratio scoring, quality subscales are divided by quantity subscales to eliminate the contaminating effects of fluency on originality (Hocevar \& Michael, 1979; Runco, 1986). However, studies have yielded mixed results with this approach, and several researchers have instead turned to subjective scoring systems.

\section{Subjective Scoring Approaches}

Subjective scoring approaches, also referred to as rater-based scoring, require 
external raters to evaluate participants' originality. These approaches represent the line of thought that ideas and actions are original if they are socially recognized as such. Though different criteria have been used for these ratings, researchers base subjective ratings on predominant definitions of originality. For example, several studies (e.g., Forthmann et al., 2017; Silvia et al., 2008b) use a Likert-scale to evaluate originality based on three criteria identified in a seminal study exploring individual differences in original ideation: uncommonness, cleverness, and remoteness (Wilson et al., 1953). Researchers have found that raters can reliably judge participants' responses by simultaneously considering these three characteristics of originality (Silvia et al., 2008b).

In some studies, raters judge each response in an ideational set independently. Though this approach is valued for its thoroughness, it can be time and energy consuming, especially with larger sample sizes and ideational pools. Instead, many studies will instruct raters to evaluate a subset of an individual's responses, or the entire ideational pool at once. One such approach is the "top-scoring" method. After completing a DT task, participants or experimenters are asked to examine an entire set of responses and to select a fixed number of responses (e.g., "top two") they perceive to be most creative (Benedek et al., 2013; Silvia et al., 2008b). Selected responses are then used to establish individual's originality scores using a fixed-rating scale (e.g., 5-point scale). Perhaps the most time-effective method to date is the "snapshot" approach introduced by Silvia et al., (2009). In this method, raters assign a single originality rating based on a participant's entire ideational pool.

While subjective approaches have been shown to mitigate the contaminating effects of quantity on quality subscales (Silvia et al., 2009), they also have the potential to 
introduce substantial error and bias. For example, the volume of work involved in subjective rating, especially with large datasets, has the potential to negatively impact inter-rater reliability (Forthmann et al., 2017). Furthermore, if experimenters are charged with assigning originality scores to participants' responses, their personal views of the construct may influence their selections. Although these methods include guidelines for assessing responses and inter-rater agreement is observed, they still invite avoidable systematic factors, such as raters' expertise (Silvia et al., 2008a), which can also negatively impact the replicability of findings.

\section{Which Approach is Superior?}

There is still considerable disagreement among DT researchers as to which approach is most reliable and valid, and there is evidence to suggest participant assessment outcomes are heavily influenced by the scoring methods selected by researchers (Park et al., 2016). Many scholars have called for more work implementing both approaches within a single study to better determine how the scoring method influences outcome (Plucker et al., 2011). While both approaches have clear strengths and weaknesses, I argue there are two significant, and largely overlooked, limitations of current DT methodology. First, current methods for scoring originality are largely based on social comparison. That is, the originality ratings of one child's responses is at least partially dependent on the ratings of other children in the sample. While this is especially true of objective scoring systems, the same can be said for subjective approaches that instruct raters to familiarize themselves with the complete ideational pool prior to assigning ratings. Second, regardless of the two general scoring approaches described above, children's DT is typically studied using cross-sectional designs rather than 
longitudinal designs. I believe these two limitations of current DT research, especially with young children, may restrict the understanding of originality in two primary ways:

(a) they limit the ability to precisely capture personal originality, and (b) they do not adequately account for the role of time.

\section{The Personal-Originality Approach}

In its most basic form, DT is thought to be a characteristic of the individual. Even if researchers implement a large battery of DT assessments, outcome measures typically do not effectively examine individual thought processes and originality. Given this, use of social comparison as the definition of creative potential will never capture whether or not an idea is original to the child, regardless of whether the researchers implement objective or subjective scoring, or both. Kaufman and Beghetto's (2009) 4-C model of creativity proposes the importance of a "mini-c" category that represents "the creativity inherent in students" unique and personally meaningful insights" and "highlights...more process focused aspects of creativity" (p. 4). In line with this thinking, I believe it is important to recognize originality that occurs at this individual and process-oriented level. Plainly stated, I believe if an idea is original for the individual, the individual is, by definition, thinking divergently.

One way to study such personal originality (PO) is the old/new approach (K. J. Gilhooly et al., 2007). That is, after participants complete a DT task, researchers can ask participants to identify which responses they had thought of prior to data collection, and which responses they generated during the DT task. This is a highly cost- and timeeffective method for gathering within-individual originality data and can be implemented in cross-sectional designs. However, it may be less effective to implement this approach 
with younger populations due to vast individual differences in memory retrieval abilities that would have to be measured and accounted for. Further, these instructions would add an additional layer of complexity to the child's experience, especially if multiple DT tasks are implemented. In turn, this would increase the overall cognitive demands of each task and, potentially, impact children's performance. An alternative PO approach is to measure how DT unfolds over time. For example, one might compare an individual's responses at one time point to future time points and operationalize $\mathrm{PO}$ in terms of new additions to the original set of responses. This approach has the added benefit of helping address the second mentioned methodological limitation, time.

\section{The Role of Time in Divergent Thinking}

Cross-sectional research does not adequately account for the role of time in divergent thinking. Within single-session studies, time has been recognized as an important factor in DT measurement. For example, researchers have found that DT tasks are less effective when participants are given a time limit compared to having no time constraint (Johns \& Morse, 1997). Researchers have also found that original ideas are more likely to appear later in a set of responses rather than earlier (Beaty \& Silvia, 2012). This suggests the more time participants have to explore an open-ended problem, the more likely they are to produce original solutions. It stands to reason a more thorough examination of the effects of time on children's DT outcomes might provide unique insights into how to best measure the construct, and possibly inform theory about the fluidity of it.

One promising time-related construct is incubation, which has been defined as the unconscious processing that occurs during "voluntary abstention" from a problem 
(Wallas, 1926, p. 86). Research with older populations has demonstrated the importance of incubation periods in the creative thinking process (Ellwood et al., 2009; K. J. Gilhooly et al., 2013; Kenneth J. Gilhooly et al., 2012; Sio \& Ormerod, 2009). In a metanalysis of 117 studies, Sio and Ormerod (2009) found that the length of preparation prior to incubation, the complexity of incubation tasks, and nature of the primary task were key factors that explained study outcomes. Specifically, incubation effects were more pronounced when (a) participants had more time to work on the problem prior to incubation, (b) incubation tasks had low cognitive demands, and (c) the primary task involved divergent thinking as opposed to tasks with target/fixed solutions.

To the best of my knowledge, the role of incubation in children's DT has not been studied directly, making it difficult to determine how to best structure incubation periods within early childhood DT assessments. However, it is reasonable to assume this dimension of the creative-thinking process extends to young children's DT and would therefore be a logical and meaningful methodological consideration. It is possible that both within- and between-session incubation periods will help illuminate how time away from a DT task influences children's performance. One way to test these alternative forms of incubation is to design studies that examine each independently. This approach would have the benefit of minimizing the effort expended by both the experimenters and participants. Indeed, if longitudinal data collection proves unnecessary for studying DT in the context of incubation, researchers could collect large amounts of meaningful data in a cost- and time-effective manner. However, without studying various lengths of incubation, including periods that demand longitudinal data (e.g., several days) within a single study, researchers will not be certain if, how, and to what extent children's 
divergent thinking processes are influenced by time off-task.

Another approach is to invest in large-scale longitudinal studies. If researchers examine DT performance while manipulating incubation over long periods of time within large samples of children, they might be able to pinpoint incubation "sweet spots" (e.g., the ideal amount of time resulting in peak performance) or even "dead zones" (e.g., too much time resulting in worsened performance). The downside of this approach is that the knowledge gained might not be worth the effort and resources expended. Thus, I believe the logical first step is to balance feasibility with scalability. A small-scale study with two time points should provide important insights into the relation between incubation and divergent thinking among young children. Should such a study yield compelling results, more ambitious longitudinal designs will be justified.

\section{Current Study}

The current study investigates two primary research questions. First, is the personal-originality approach a more valid method for capturing young children's DT compared to common objective and subjective approaches? To explore this question, an author-developed, personal-originality scoring system was used to measure children's DT performance. The current study compares the construct validity of objective (i.e., weighted ratio scores), subjective (i.e., average external ratings), and PO scoring methods by correlating children's DT outcomes with individual characteristics (e.g., openness to experience) and performance measures (e.g., creativity ratings of children's drawings) commonly identified as indicators creative potential. Originality is often considered the most creativity-specific subscale of DT (Runco \& Acar, 2012). Therefore, this study focuses on the validity of originality outcomes across various scoring methods. A 
detailed description of all scoring methods used in the current study can be found in Appendix A. Two competing hypotheses were tested to address the first research question:

H1: PO scoring for originality will provide a more valid estimate of children's creative potential compared to subjective and objective scoring. PO scores demonstrating significantly better measures of convergent and discriminant validity compared to traditional scoring methods would provide support for this hypothesis.

$\mathrm{H} 2: P O$, subjective, and objective ratings of originality all represent meaningful, yet distinct, constructs related to children's overall creative potential. Said differently, children who produce ideas that are (a) original at a personal level (PO), (b) unique in the sample (objective), and (c) recognized as creative by outsiders (subjective) might be among the most creative. If this is true, the three measures will yield comparable estimates of convergent validity with other measures of creative potential but would show discriminant validity between the three originality outcomes.

The second research question investigated in the current study was do short and/or long incubation periods help illuminate children's original ideation processes? Due to the exploratory nature of this research question, the following hypothesis was tested:

H3: Within-session incubation periods will provide optimal conditions for childlevel original ideation to occur. It is my thinking that briefly reallocating children's focus to an unrelated task will allow sufficient time for incubation and subsequent original ideation to occur. In contrast, between-session incubation may result in difficulty recalling previous task performance, thereby making it challenging for children to focus 
their effort on producing new ideas. In addition to a visual inspection of descriptive data and an examination of correlations between short and long PO scores and other outcome variables, dependent $t$-tests were used to detect any significant differences between short and long PO scores.

\section{A Note on the COVID-19 Pandemic}

Data for the current study were collected during the COVID-19 pandemic. To ensure the safety of children and caregivers, all aspects of the study were conducted remotely. While parent data are often collected in this manner (e.g., online surveys), I recognize that remote data collection with young children is uncommon. Though this was not the topic of the current study, I believe it is important to note that children were highly engaged in study tasks and excited to interact via video conference software. The implications of remote data collection are apparent in the method section and outlined explicitly in discussion. Ultimately, the decision to prioritize safety measures was an easy one to make and I am pleased with how the study unfolded. 


\section{CHAPTER 2: METHODOLOGY}

The purpose of the current study was to explore a new incubation-based, personal-originality approach for scoring children's divergent thinking and to compare this scoring system to common objective and subjective approaches. Based on the overarching goals of the study, and limitations caused by the COVID-19 pandemic, a non-experimental, longitudinal, and correlational design was used.

\section{Recruitment and Participants}

To maximize the sample for this study, online recruitment and snowball sampling were used. Recruitment ads were disseminated through university-affiliated listservs, emails to preschool directors, and social media. 57 parents initially responded to recruitment ads. Of those, 42 parents participated in the initial survey and enrolled their children in the study. The vast majority of parents (93\%) who completed surveys and accompanied children during zoom sessions self-identified as the mother of the enrolled child. 42 children $(64 \%$ female $)$ between the ages of 3-6 $(M=4.84$ years; $S D=1.21)$ completed the zoom session. Two parents did not complete the follow-up survey. After additional attempts to collect follow-up survey data from these parents, both disclosed sensitive circumstances preventing them from engaging in non-essential time commitments. All parents were entered in a lottery to receive one of ten $\$ 25$ gift cards for participating in the study.

\section{Procedure and Measures}

\section{Overview}

A two-part parent survey was used to gather information about children's divergent thinking skills, creativity, personality, and background information about the 
parent, child, and family. Part 1 of the survey was distributed upon enrollment in the study through the University of Missouri Qualtrics System. After completing part 1, parents scheduled a zoom session for their enrolled children. Detailed instructions regarding the zoom session were sent 48 hours prior to the scheduled date. Parents were asked to position their children in a comfortable, quiet, and distraction-free environment, and to remain present with their children throughout the session. Parents were encouraged to redirect children's attention to the study tasks as needed but to offer no other assistance. All zoom sessions were video- and audio-recorded with parent consent. 48 hours after the zoom session, part 2 of the survey was sent to parents. Specific measure items and detailed instructions are presented in Appendix B. Detailed descriptive properties for all variables listed below are presented in Table 1.

\section{Parent Survey Part 1}

Child Demographics. At the beginning of the survey, parents were asked to write in demographic information including children's age, gender, race, and ethnicity. Age was coded as a continuous variable and was calculated using the exact number of days between date of birth and the child's zoom session. Gender was coded as male or female (female $=1$ ). Due to minimal racial and ethnic diversity $(79 \%$ were identified by parents as "White") in the current study, children were classified as White or Racial-Ethnic Minority (White = 1).

Big Five Inventory - Short Form. The Big Five Personality Inventory - Short Form (BFI-10; Rammstedt \& John, 2007) is an efficient tool for measuring children's personality traits. It is an abbreviated version of the 44-item tool and consists of 2 items per scale, for a total of ten items. This tool prioritizes speed and ease of administration 
while still maintaining acceptable levels of reliability and validity. The BFI-10 was primarily used to capture children's openness to experience and extraversion, both of which are theoretically and empirically linked to creative potential (Furnham \& Bachtiar, 2008; Gocłowska et al., 2019; McCrae, 1987).

Parent Rating of Child Creativity. Parents were asked to rate their children's creativity on a scale from 1 (not at all) to 10 (extremely) to achieve a subjective rating of child creativity prior to child zoom sessions.

\section{Zoom Session Tasks}

After a brief period of introductions at the beginning of the recorded zoom session, children completed a total of seven tasks. Apart from the first (i.e., define creativity) and last (i.e., verbal reasoning) tasks, task order was randomized. Two variables were created to account for potential task order effects: (a) drawing first (yes = 1) indicates whether children completed the drawing before or after DT tasks and (b) instances first (yes $=1$ ) was used to identify the order DT tasks were presented to children. In addition, an incubation length variable was created to determine if the amount of time (in days) between the zoom session and follow-up DT tasks influenced personal-originality scores.

Define Creativity. Children were asked if they had ever heard the word "creative" or "creativity." Those who nodded or verbally stated they had heard of either word were asked if they knew what the word meant and, if so, to describe it. Accurate

definitions (e.g., "doing something new") were assigned a " 1 " and inaccurate responses/no response was scored " 0 ." Children were then given a formal definition of creativity prior to transitioning into the primary tasks. 
Creative Drawing Task. Children were asked to think of something creative to draw and, after completing the drawing, children were asked to think of a creative name or title for their drawing. To establish a performance measure, drawings and titles were each rated on a scale from 1 (not at all creative) to 5 (extremely creative) by two independent raters using the criteria outlined in Appendix C. These two scores were added to create an overall drawing/title score. An example of a child's drawing with its corresponding title is presented in Figure 1.

Divergent Thinking. Two divergent thinking tasks were included in the current study: Wallach and Kogan's (1965) instances task (i.e., things that are round) and Guilford's (1967) alternative uses task (i.e., uses for a pencil). To introduce the instances task, a picture of a marble was displayed on the screen and a physical marble was held up to the camera. To introduce the alternative uses task, a picture of a pencil was displayed on the screen and a physical pencil was held up to the camera. Images depicting the zoom session screen share during DT tasks are displayed in Figure 2 and Figure 3. Children completed each task twice during the session (before and after incubation) and again when parents completed the follow-up survey. Because time limits can negatively affect DT performance (Johns \& Morse, 1997), children were given unlimited time to complete each task. A complete list of children's responses for each DT task are outlined in Appendix D.

Filler Tasks. Two filler tasks were used to create within-session incubation periods. After children indicated they had exhausted their ideas for a given DT task, they were presented a series of simple shapes (i.e., square, heart, triangle, and star) filled in with red, blue, green, orange, and pink were presented one at a time for two minutes. 
During one incubation period, children were instructed to identify the shape and in the other, children identified the color. These mundane filler tasks were selected based on the Sio and Ormerod (2009) finding that incubation tasks with low cognitive demands are most effective. The duration of the incubation period was based on findings that 2-minute incubation periods yield significant incubation effects while shorter periods (e.g., 30 seconds) do not (Smith \& Blankenship, 1991).

Verbal Reasoning. Children completed five items of a guessing game adapted from the Guess What task of the Reynolds Intellectual Assessment Scales (Reynolds \& Kamphaus, 2005). This task is designed to measure verbal reasoning and crystallized intelligence, which are established covariates of verbal divergent thinking performance (Jones \& Estes, 2015; Weiss et al., 2020). Children are instructed to carefully listen to three clues and then guess what the clues describe. For example, an accurate response for the three hints "it falls from the sky, it helps plants grow, and if it falls on you it makes you wet" would be "rain." Children received one point for each accurate response for a total possible score of five points.

\section{Parent Survey Part 2}

Follow-Up DT. Parents were asked to complete a follow-up session of the instances and alternative uses task in one sitting. A script was provided for reintroducing (e.g., "Do you remember when you played a game on the computer where a man showed you a marble and asked you to think of things that are round?") and providing instructions (e.g., "Can you think of anything else that is round? Try to be as creative as possible!") for each task. Parents recorded their children's responses and, after children 
indicated they had no more ideas, parents submitted the complete list via Qualtrics survey.

\section{Divergent Thinking Scoring Approaches}

Prior to scoring, the entire pre-incubation ideational pool was compiled and alphabetized with and without identifiers. Figure 4 illustrates how identical and near identical responses were treated for valid scoring. Fluency scores for instances and alternative uses were measured as the total number of unique responses produced prior to within-session incubation. In addition, four originality scores for instances and alternative uses were produced for each child: objective, subjective, short PO, and long PO (see Appendix A).

Objective Scoring. A combination of weighted and ratio scoring techniques were used to produce one instances and one alternative uses objective score using only responses produced prior to the within-session incubation period. 1 point was assigned for responses produced by $<10 \%$ of children and 2 points were assigned for responses produced by $<5 \%$ of children. All other responses received 0 points. Research has demonstrated that this type of weighting improves the validity of originality scores (Runco et al., 1987).The total originality score was then divided by the number of ideas produced (i.e., ratio scoring) to mitigate fluency contamination (Hocevar \& Michael, 1979).

Subjective Scoring. Two raters used the uncommon, remote, and clever criteria outlined in Silvia et al. (2008), and presented in Appendix C, to score each response in the ideational pool on scale from 1 (not at all creative) to 5 (highly creative). To control for fluency, participants' total subjective scores were divided by fluency to produce one 
instances and one alternative uses subjective score using only responses produced prior to the within-session incubation period.

Personal-Originality Scoring. Short PO scores were generated by comparing responses produced before filler tasks to those produced after filler tasks. One point was assigned for each new response to produce one instances and one alternative uses short PO score. Long PO scores were generated by comparing responses produced in the follow-up survey to all previous responses. Again, one point was assigned for new responses for each DT task.

\section{Data Analysis Plan}

To address the first research question, the current study compared the convergent and discriminant validity of scoring methods using standard correlational analyses utilized in previous DT measurement validity research (Benedek et al., 2013; Clapham, 2004). Convergent validity was evaluated by exploring the associations between various scoring approaches and established covariates of divergent thinking (e.g., openness to experience) as well as performance measures of creativity (i.e., creative drawings and titles). Discriminant validity was analyzed by examining correlations among PO, objective, and subjective outcomes, as well as between each scoring method and taskspecific fluency scores.

To explore the second research question comparing the effectiveness of short (within-session) vs. long (between-session) incubation periods, descriptive and correlation analyses were conducted in addition to means difference testing. Paired sample $t$-tests were conducted to detect significant differences between short and long personal-originality score means for instances and alternative uses tasks. 
Finally, a set of preliminary and post-hoc analyses were conducted to explore individual characteristics (e.g., child age) and external factors (e.g., task order) that might explain differences in personal-originality outcomes. For post-hoc analyses, a series of bivariate and multiple regression models were conducted with personal-originality scores for instances and alternative uses tasks as dependent variables and correlates revealed during preliminary and primary analyses as independent variables. Next, hierarchical regression analyses were conducted to examine the effects of PO scores on children's combined drawing/title creativity ratings while controlling for relevant child characteristics identified in preliminary and primary analyses. 


\section{CHAPTER 3: RESULTS}

\section{Preliminary Analyses}

Preliminary analyses were conducted to determine which demographic and study variables should be included in primary analyses. Zero-order bivariate Pearson and pointbiserial correlations revealed that gender, race and ethnicity, drawing first, and instances first were not associated with any DT outcomes, nor performance measures of creativity, and were therefore omitted from primary analyses. Furthermore, despite there being considerable variation in the length of between-session incubation $(M=6.33$ days; $S D=$ 4.55; Range $=2$ to 22 days), incubation length was not significantly correlated with AUT $(r=.012, p=.94)$ nor IT $(r=-.17, p=.29)$ long PO scores. Statistically significant correlations were, however, identified between age and several performance measures. Therefore, a detailed exploration of age effects was included in post-hoc analyses outlined at the end of the results section.

In addition, intraclass correlations (ICC) and 95\% confidence intervals, reported in Table 2, were used to assess the inter-rater reliability of two independent ratings for subjective instances, subjective alternative uses, creative drawings, and creative titles. Since the same raters were used across all subjects and tasks, two-way mixed effects models using average measures and absolute agreement were used (Shrout \& Fleiss, 1979; McGraw \& Wong, 1996). To briefly summarize, ICCs for subjective instances, subjective alternative uses, creative drawings, and creative titles were $.747, .813, .740$, and .775 , respectively. All ICCs were between the "moderate" and "good" range according to Koo and Li's (2016) guidelines. Therefore, the averages of independent ratings for these variables were used in primary analyses. 


\section{Primary Analyses}

Primary analyses are organized into two sections: (a) correlational analyses detailing the associations among originality scores and primary variables, and (b) exploratory analyses of incubation effects.

\section{Correlation Analyses}

As previously stated, correlational analyses were used to compare and contrast the convergent and discriminant validity of originality outcomes. Zero-order bivariate Pearson and point-biserial correlations for primary analyses variables are presented in three tables. Table 3 shows correlations among primary variables and DT instances task scores, Table 4 shows correlations among primary variables and DT alternative uses task scores, and Table 5 shows correlations between DT instances (IT) and DT alternative uses (AUT) scores. Based on the current sample size, statistically significant correlations between theoretically linked constructs were considered evidence of convergent validity, while non-significant correlations among theoretically distinct constructs were considered evidence of discriminant validity.

Convergent Validity. The current study failed to demonstrate correlations between originality outcomes and personality traits associated with divergent thinking. However, this is at least partially explained by the minimal variance in parent ratings of openness to experience and extraversion. All ratings for extraversion ranged between 3 and 6 despite the scale ranging from 0 to 10, and while the overall range of scores for extraversion was greater, approximately $70 \%$ of children were rated 6 or 7 . As a result, the remaining comparison of convergent validity between objective, subjective, and PO scoring is limited to parent ratings of child creativity, children's understanding of 
creativity (i.e., ability to define the construct), verbal reasoning, and performance measures of creativity (i.e., drawings and titles).

Objective originality, using weighted ratio scores, performed poorly in the current study. Objective originality was not significantly correlated with parent ratings of creativity, children's understanding of creativity, performance measures of creativity, nor verbal reasoning. Further, objective originality scores for instances and alternative uses were not significantly related. It should be noted that other approaches to objective scoring (e.g., weighted only, ratio only, total) were tested and did not produce any observable differences in evidence for convergent validity. Previous research has suggested objective originality scoring is most effective with larger sample sizes (e.g., $N$ $=300-400$; Forthmann et al., 2020), and it is possible that my sample of 42 children was not sufficiently large for a "statistical rarity in the sample" definition of originality to perform well. Despite this, findings from the current study suggest objective scoring has poor convergent validity.

In comparison to objective scoring, subjective originality scoring performed better and demonstrated some evidence of convergent validity. Subjective originality scores, based on the average score of two independent raters and divided by fluency, were significantly associated with children's drawing and title creativity ratings, as well as verbal reasoning scores, with Pearson correlations between .37 and .44 . However, these associations were only apparent using DT scores from the alternative uses task. IT subjective ratings, despite being significantly associated with AUT scores $(r=.38)$, were not significantly correlated with any primary study variables apart from other DT outcomes. It is not uncommon in DT research for analyses to reveal task-specific effects, 
which has led to theoretical discussions about the potential domain specificity of divergent thinking (Baer, 2012; Han, 2003). That aside, evidence of convergent validity for subjective originality scores was related to the specific divergent thinking task.

Overall, personal-originality scoring, and particularly short PO scores, had the most compelling evidence for convergent validity. PO scoring using the long-incubation technique (i.e., between sessions) showed similar trends as subjective scoring. Long PO scores for the instances task were associated with performance measures of creativity and verbal reasoning, but no significant correlations were revealed for alternative uses task long PO scores. Short PO scores across DT tasks were significantly correlated with parent ratings of creativity, creative drawings and titles, and verbal reasoning. In addition, short PO scores were the only originality outcomes associated with children's ability to define creativity. Both short and long PO showed strong, positive, between-task correlations, $r=.53$ and $r=.62$, respectively. Altogether, current study findings indicate that personal-originality scores after a short (i.e., 2 minute) incubation period have high convergent validity, long PO and subjective ratings of originality have task-dependent convergent validity, and objective ratings have poor convergent validity.

Discriminant Validity. Discriminant validity was evaluated by examining correlations (see Table 5) among task-specific originality scoring approaches and between task-specific originality and fluency scores. Objective, subjective, and personal originality represent distinct theoretical views of originality and should, therefore, be minimally correlated. Similarly, originality and fluency are thought of as independent dimensions of divergent thinking. 
While objective scoring showed low convergent validity, it demonstrated the greatest evidence for discriminant validity. Objective originality was not correlated with fluency in either task, and the only significant correlations between objective scores and other measures of originality were between AUT objective and subjective scores and IT objective and long PO. This finding suggests weighting originality and dividing the total score by fluency helped prevent contamination and promoted discriminant validity in the current study.

Similar to findings about convergent validity, evidence for the discriminant validity of subjective ratings was task specific. IT subjective scores showed strong discriminant validity with no significant correlations with other IT outcome measures. However, AUT subjective scores showed significant positive correlations with objective, short PO, and fluency scores, indicating poor discriminant validity in this task.

Long personal originality showed similar levels of discriminant validity across tasks. AUT long PO scores were positively correlated with fluency, but not other originality scores. IT long PO scores significantly related to fluency and short PO, but not subjective nor objective originality. Though evidence for short PO discriminant validity is implicitly stated previously in this section, it can be summarized as follows: both short PO scores were significantly associated with fluency and one other originality score, within-task. Though this evidence collectively suggests poor discriminant validity for short PO, and moderate task-specific discriminant validity for long PO, these findings warrant further investigation with larger and more diverse samples.

\section{Incubation Effects}

As can be seen in Table 1, children's short and long PO scores are relatively 
comparable within each task. For IT, children generated between 0 and 13 responses ( $M$ $=4.69)$ after short incubation and 0 and 16 responses $(M=5.43)$ after long incubation. Paired samples $t$-tests revealed no significant differences between IT short and long PO means $t(39)=-0.844, p=.40$. For AUT, children generated between 0 and 9 responses $(M=2.64)$ after short incubation and 0 and 8 responses $(M=2.10)$ after long incubation. Again, means differences between short PO and long PO scores were not statistically significant $t(39)=1.521, p=.14$. There were, however, significant differences in PO performance across tasks. Children produced significantly more new ideas after short incubation during the IT compared to the AUT, $t(41)=4.504, p<.001$. Similarly, children produced significantly more new responses after long incubation for the IT compared to AUT performance $t(39)=8.645, p<.001$. Given these between-task differences in short and long personal-originality scores, it is interesting to note that DT task order was not significantly related to performance.

\section{Post-hoc Analyses}

Based on correlational analyses, only three personal-originality outcomes warranted further exploration. IT short and long PO scores, and AUT short PO scores demonstrated significant associations with primary study variables. A series of multiple regression models with PO scores as dependent variables and identified correlates as independent variables are presented in the following paragraphs. As previously stated, age was revealed as the only child demographic factor significantly correlated with DT outcomes and child performance measures and was therefore included in all regression analyses. Fluency and verbal reasoning scores were correlated with all dependent 
variables and were included stepwise, based on correlation strength, to identify the bestfitting models using $F$-Change statistics.

\section{Linear Regression Assumptions Testing}

The linear relation between independent variables and dependent variables was verified through a visual inspection of scatterplots. Extreme outliers were identified through visual inspection of boxplots, and standardized residuals $+/-3$ were removed prior to each set of analyses (Field, 2009). Evidence that assumptions for multicollinearity, normal distribution of residuals, and homoscedasticity were not violated are presented in Appendix E.

Post-Hoc Analysis Results

Overall model results are briefly described in this section. Unstandardized beta coefficients, standard errors, and standardized coefficients for individual predictors in each model are presented in Table 6. The best fitting model for alternative uses task short PO and subjective ratings included age and fluency as independent variables. Age and AUT fluency explained $71.7 \%$ of the variance in AUT short PO $F(2,36)=45.57, p<$ .001 . This finding indicates that within-session personal-originality scores increase linearly with age and fluency.

The best fitting model for short PO in the instances task included only age as an independent variable, which explained $26.4 \%$ of variance in IT short PO, $F(1,40)=$ $14.33, p<.05$. As was the case for the alternative uses task, children's within-session personal originality for the instances task had a positive linear relationship with age. Though age was not a significant predictor of IT long PO, its inclusion in the model did not change model summary results and was therefore included for consistency. Age and 
IT fluency explained $24.4 \%$ of variance in long PO scores, $F(2,37)=5.98, p<.05$. In this case, increases in ideational output (i.e., fluency) were associated with increases in between-session personal-originality scores for the instances task.

Finally, hierarchical regression analyses were conducted with the combined drawing/title creativity ratings as the dependent variable. Children's age was included as an independent variable in block one for all models due to its strong significant association with the dependent variable. Three separate models were conducted with AUT short PO, IT short PO, and IT long PO included as block two independent variables. A separate model for AUT long PO was not conducted because it was not significantly correlated with the dependent variable. Results, presented in Table 7, indicate that AUT short PO scores did not significantly predict combined drawing/title ratings when controlling for age, and did not significantly improve the fit of the model that only included age as predictor. However, both IT personal-originality scores were significant predictors of combined drawing/title ratings when controlling for age.

The inclusion of long PO significantly improved model fit and resulted in an $R^{2}$ change of .159 (from .229 to .388), indicating the model including both age and long PO explained $15.9 \%$ more variance in children's combined drawing/title scores compared to the model that only included age. Similarly, the addition of short PO significantly improved model fit and resulted in an $R^{2}$ change of .11 (from .214 to .323 ), which suggests the second model explained $11 \%$ more variance in children's combined drawing/title scores. After a visual inspection of regression coefficients in the IT short PO model, follow-up bootstrapping tests were conducted and indicated full mediation (see Figure 7). The total effect of age on combined drawing/title scores in model 1 was 
statistically significant, $B=.863, t(40)=3.30, p<.05$. However, the direct effect of age after including IT short PO was no longer statistically significant, $B=.493, t(39)=1.72$, $p=.09$, while the direct effect of IT short PO was statistically significant, $B=.253, t(39)$ $=2.51, p<.05$. This finding suggests that the relation between age and creative drawing/title ratings is explained by within-session personal-originality scores for the instances task. 


\section{CHAPTER 4: DISCUSSION}

The discussion of the current study is organized in five sections. The first two sections address study research questions and hypotheses. In section one, I discuss empirical support for the construct validity of each originality scoring approach used in the current study. In section two, I contextualize findings about the effects of short and long incubation periods on children's personal originality. Next, I interpret the results of post-hoc analyses and discuss child characteristics associated with divergent thinking outcome measures. Fourth, I briefly discuss my experience collecting data remotely and the implications of this "contactless" method of data collection with young children. Finally, I outline study limitations and implications, and present ideas for future research based upon my findings.

\section{Evidence for Construct Validity}

The primary objective of the current study was to address the research question is the personal-originality approach a more valid method for capturing young children's DT compared to common objective and subjective approaches? Previous research testing the convergent validity of originality outcomes has analyzed correlations among divergent thinking scores and other established indicators of creative potential. The current study followed this approach and investigated associations between objective, subjective, and PO originality scores, child characteristics (i.e., personality, parent ratings of child creativity), performance measures of creativity (e.g., creative drawings), and verbal reasoning skills.

Based on the findings of the current study, personal-originality scoring produced considerably better evidence of convergent validity compared to subjective and objective 
approaches. This was especially the case for personal-originality scores after withinsession incubation periods (i.e., short PO). Conversely, personal-originality scoring showed poor evidence of discriminant validity when compared to objective scoring, and similarly levels of discriminant validity when compared to subjective scoring. However, if correlations between short and long PO scores are not considered, which is reasonable due to conceptual overlap, the primary distinguishing feature between personaloriginality scoring and objective scoring, in terms of discriminant validity, are correlations with fluency. The relation between PO and fluency is intriguing. Intuitively, one might expect a negative correlation between these constructs (e.g., exhausting background knowledge prior to incubation could negatively impact original ideation post incubation). Instead, generative ability prior to incubation was positively related to original ideation after incubation in the current study. Though this finding does not support the discriminant validity of incubation-based PO scoring in the current study, it does serve as an interesting topic for future research.

Neither set of a priori criteria established for hypothesis 1 ( $P O$ is a more valid indicator of creative potential), nor hypothesis 2 (all three originality scores are meaningful), were fully satisfied in the current study. However, the compelling evidence for the convergent validity of personal-originality scoring warrants further consideration in the methodological and theoretical discussions surrounding children's divergent thinking.

\section{The Role of Incubation in Children's Divergent Thinking}

The second research question was do short and/or long incubation periods help illuminate children's original ideation processes? I offered the exploratory hypothesis 
that within-session incubation periods would "provide optimal conditions" for divergent thinking and original ideation to take place. Descriptive statistics and paired-samples $t$ tests did not support this hypothesis. Overall, children produced a similar number of new ideas after short (e.g., within session) and long (e.g., between session) incubation periods. As previously demonstrated, however, short PO scores were strongly associated with variables theoretically linked with divergent thinking, and long PO scores were comparable to subjective scores in that regard.

The current study findings suggest that short and long incubation periods do, indeed, "help illuminate children's original ideation processes." The incubation-based PO approach provides useful insights into the role of time in children's divergent thinking. Specifically, it shows: (a) time off task has a positive effect on children's ability to produce new ideas, and (b) that a single set of responses provides an incomplete picture of children's DT. It should be noted that incubation did not result in original ideation for all children. For the alternative uses task, nine children did not produce new ideas after short incubation, and eight children did not produce new ideas after long incubation. For the instances task, the number of children who did not produce new ideas after short and long incubation were four and one, respectively. Altogether, findings support the continued use and exploration of incubation periods in child DT research.

\section{Child Characteristics and Divergent Thinking Scores}

Post-hoc analyses revealed that age and task-specific fluency scores explained significant variance in several originality outcome measures. I have previously discussed the interesting finding that fluency scores were positively correlated with personaloriginality scores. This was also true for AUT subjective scores, which does not align 
with previous research demonstrating negative correlations between average subjective ratings and fluency (Benedek et al., 2013). However, correlations between fluency and originality scores ranging from .34 to .56 are not uncommon and can be expected given their joint association with a shared construct.

I believe the more interesting child characteristic to investigate further is age. Though the wide range of ages represented in the current study poses some issues, it also provided useful insights into the developmental nature of divergent thinking. Figures 5 and 6 help illustrate how age, transformed into a categorical variable grouped by year, relates to divergent thinking outcomes. Mean objective, subjective, and long PO scores are relatively stable across age groups for both instances (Figure 5) and alternative uses (Figure 6). However, interesting developmental trends emerge for fluency and short PO scores. Fluency mean scores were consistent for children between 3-5 years and increased dramatically among 6-year-olds, suggesting an increase in ideational output for the oldest age group. Short PO scores, on the other hand, showed a gradual increase between 3-6 years. Though this finding should be interpreted and generalized tentatively, the current study suggests that as children get older, within-session incubation effects have a more profound effect on original ideation. Furthermore, these trends appear to be independent of developmental trends in pre-incubation fluency.

Finally, despite considerable variance in personal originality being explained by age and strong positive correlations between age and children's combined drawing/title scores, post-hoc analyses revealed that PO scores for the instances task predicted children's performance measure of creativity above and beyond age. Moreover, short PO scores mediated the relation between age and combined drawing/title creativity ratings. 
These preliminary findings suggests that personal originality is an important consideration in the identification of creative potential.

\section{Collecting Child Data Remotely}

As previously stated, the current study was conducted during atypical times. Safety precautions, including remote data collection, were necessitated due to the COVID-19 pandemic. I should note that previous research with older populations has demonstrated the reliability of online DT assessment (Hass, 2015), but to the best of my knowledge, this is the first study that has collected child DT data remotely. There were several strengths of this "contactless" approach to child data collection, but also drawbacks that must be acknowledged. Perhaps the greatest advantages of this approach were flexibility and efficiency. Data collection with young children is often restricted to the normal operating hours of childcare centers and schools. Collecting data via zoom afforded parents considerable flexibility in terms of scheduling, including morning, afternoon, and evening options, and both weekday and weekend availability. Several parents explicitly mentioned their appreciation for this convenience.

The efficiency of remote data collection also became quite apparent. Under typical (e.g., in person, lab setting) conditions, the transition from one session to the next includes unproductive factors, such as the time it takes to locate a child enrolled in the study, help them transition from the classroom to assessment room, and return them to the class upon completion. In the current study, parents had their children prepared to engage in the study at a predetermined time, and the transition from one session to the next was seamless and predictable. In addition, there were no limitations nor scheduling issues related to assessment space. Zoom sessions lasted approximately 20 minutes, and 
recordings could be processed in 10 minutes or less. During that processing time, I was able to reset all materials and prepare for the next scheduled session without any additional concerns. With additional experimenters, the efficiency of remote data collection could be maximized. These advantages cannot be overstated, but there were disadvantages of remote data collection, as well.

Two key disadvantages became evident during data collection. The first was related to dependence on stable internet connections. There were instances when data collection had to be rescheduled due to inclement weather. Fortunately, there was never a case when a zoom session was disrupted by weather conditions in the current study, but researchers considering remote data collection should be aware of this possibility. Second, there are uncontrollable differences in children's environments. Despite parents being instructed to prepare a "quiet and distraction-free" space for children to complete the study, these environments were not identical. Controlled lab settings are not necessarily "distraction free," but they likely have fewer possible, and more consistent, distractions compared to the range of disturbances (e.g., dog barking, doorbell ringing) children might experience during remote data collection.

Differences in study environments also provide different visual cues that may influence performance on divergent thinking tasks. However, given the overarching goal of the current study, to test a personal-originality approach, it is important to recognize that these between-child differences are less likely to threaten my within-subject measure of originality compared to between-subjects measures. It is undisputable that children may have access to visual stimuli that provide advantages in terms of objective and subjective ratings (e.g., uncommon items in the room), but personal originality requires 
thinking beyond readily accessible responses. This distinction provides additional support for the PO approach, especially under conditions (e.g., remote data collection) where environmental differences in the setting of data collection are a potential concern.

\section{Limitations, Implications, and Future Directions}

\section{Limitations}

In addition to previously mentioned limitations, there are two others that I believe warrant further discussion. First, the external validity of the study is constrained by several factors. The unique sociohistorical conditions and their impact on the current study (e.g., convenience sampling, non-traditional data collection methods) should not be overlooked. Further, the relatively small sample and homogeneity of children, both in terms of key demographics (i.e., minimal racial and ethnic diversity) and internal characteristics (e.g., extraversion) restricts the generalizability of findings. For example, the current study was unable to replicate well-established associations between divergent thinking and key personality traits, and while variance issues were likely the root cause of this non-finding, it is impossible to determine if this was a measurement issue, a sampling issue, or both.

The second major limitation is the questionable reliability and validity of long PO scores. In order to minimize the burden on parents and promote participant retention, I elected not to conduct a second zoom session. Instead, long PO scores were based on divergent thinking data collected by parents. Though clear instructions for conducting DT tasks were provided (e.g., "Please feel free to offer some encouragement and help keep your child focused on the task, but do not help your child come up with answers."), I cannot say with certainty that parents did not provide varying degrees of assistance. 
Further, it is impossible to account for all individual differences in experiences between the zoom session and follow-up DT assessment. However, this would also be the case had I conducted a second child zoom session. Finally, the varying lengths of betweensession incubation should be acknowledged. Despite no significant association between incubation length and long-PO scores, it is likely that the amount of time that passed between the zoom session and follow-up DT assessment had at least some impact on children's task performance. Future research should emphasize the systematicity of between-session incubation to improve the validity of findings. All limitations aside, I believe the current study offers several methodological and theoretical contributions.

\section{Implications and Future Directions}

The primary innovation of the current study was the use of incubation periods as a device for studying personal (i.e., within-subject) originality. My findings indicate that the personal-originality approach is comparable to, and in some cases better than, the subjective and objective scoring methods used in terms of construct validity. Further, I would be remiss if I did not mention the practicality and efficiency of PO scoring. For example, compared to independently rating each response in an ideational pool, repopulating those ratings to child identifiers, and producing an average rating based on fluency scores (i.e., average subjective ratings), incubation-based PO can be quickly calculated using simple excel formulas to compare columns and identify new responses at the child level. Also, the time and energy required to calculate PO scores is independent of sample size, which cannot be said for the objective and subjective approaches used in the current study.

There were also significant theoretical contributions of the current study. First, 
incubation appears to be just as important for children's original ideation as it is for adults. Current study findings suggest both short (2 minutes) and long (several days) breaks from divergent thinking, initiated only after children have exhausted their available ideas, promote future original ideation in that domain. In addition, PO outcome measures showed strong between-task correlations, which provides initial evidence for the potential domain-generality of personal originality. I believe this speaks to the individual and internal processes responsible for divergent thinking and, when studied in this manner, findings more closely approximate real-world divergent thinking. Second, the compelling evidence for convergent validity suggests child-level originality is a meaningful dimension of children's divergent thinking. Though I did not establish conclusive evidence that all three scoring approaches highlight unique dimensions of creative potential, I believe future research will support that hypothesis. Considering one of the primary goals of DT assessment is to identify and support children's creative potential, it stands to reason that both social comparison (e.g., average subjective ratings) and self comparison (i.e., personal originality) measures of originality should be evaluated.

Based on the findings and limitations of the current study, I believe there are several key considerations for future research. First, a larger and more diverse sample of children should be studied to replicate findings and improving upon the validity of my incubation-based, personal-originality scoring system. Second, based on the advantages of remote data collection, future research should compare the effects of in-person versus remote data collection on children's DT outcomes. Third, the personal-originality approach should be tested with both verbal and non-verbal (e.g., figural) divergent 
thinking tasks in a single study.

Regarding incubation effects, there are also many methodological considerations and topics to explore. For example, between-session incubation effects should be assessed under the same conditions as within-session incubation to improve measurement validity and reliability. In addition, various lengths of short (e.g., 2-10 minutes) and long (e.g., 5-24 hours) incubation, as well as different types (e.g., immediate vs. delayed) of incubation should be thoroughly and systematically studied with children. Finally, I believe a large-scale longitudinal examination of divergent thinking using short and long incubation periods would provide useful insights into the developmental nature of personal originality. Though the current study revealed intriguing developmental trends in fluency and personal originality based on sample means, it would be more enlightening to examine child-level developmental trajectories. A deeper understanding of individual differences in personal originality and how it develops would help inform parenting and educational practices intended to promote divergent thinking skills. 


\section{REFERENCES}

Baer, J. (2012). Domain Specificity and the Limits of Creativity Theory. The Journal of Creative Behavior, 46(1), 16-29. https://doi.org/10.1002/jocb.002

Beaty, R. E., \& Silvia, P. J. (2012). Why do ideas get more creative across time? An executive interpretation of the serial order effect in divergent thinking tasks. Psychology of Aesthetics, Creativity, and the Arts, 6(4), 309-319. https://doi.org/10.1037/a0029171

Benedek, M., Borovnjak, B., Neubauer, A. C., \& Kruse-Weber, S. (2014). Creativity and personality in classical, jazz and folk musicians. Personality and Individual Differences, 63, 117-121. https://doi.org/10.1016/j.paid.2014.01.064

Benedek, M., Mühlmann, C., Jauk, E., \& Neubauer, A. C. (2013). Assessment of divergent thinking by means of the subjective top-scoring method: Effects of the number of top-ideas and time-on-task on reliability and validity. Psychology of Aesthetics, Creativity, and the Arts, 7(4), 341-349.

https://doi.org/10.1037/a0033644

Cheung, R. H. P. (2012). Teaching for creativity: Examining the beliefs of early childhood teachers and their influence on teaching practices. Australasian Journal of Early Childhood, 37(3), 43-52. https://doi.org/10.1177/183693911203700307

Clapham, M. M. (2004). The Convergent Validity of the Torrance Tests of Creative Thinking and Creativity Interest Inventories. Educational and Psychological Measurement, 64(5), 828-841. https://doi.org/10.1177/0013164404263883

Cramond, B., Matthews-Morgan, J., Bandalos, D., \& Zuo, L. (2005). A report on the 40year follow-up of the torrance tests of creative thinking: Alive and well in the new 
millennium. Gifted Child Quarterly, 49(4), 283-291.

https://doi.org/10.1177/001698620504900402

Ellwood, S., Pallier, G., Snyder, A., \& Gallate, J. E. (2009). The incubation effect:

Hatching a solution. Creativity Research Journal, 21(1), 6-14.

https://doi.org/10.1080/10400410802633368

Field, A. (2009). Discovering Statistics Using SPSS. SAGE Publications. https://books.google.com/books?id=a6FLF1YOqtsC

Forthmann, B., Holling, H., Zandi, N., Gerwig, A., Çelik, P., Storme, M., \& Lubart, T. (2017). Missing creativity: The effect of cognitive workload on rater (dis)agreement in subjective divergent-thinking scores. Thinking Skills and Creativity, 23, 129-139. https://doi.org/10.1016/j.tsc.2016.12.005

Forthmann, B., Paek, S. H., Dumas, D., Barbot, B., \& Holling, H. (2020). Scrutinizing the basis of originality in divergent thinking tests: On the measurement precision of response propensity estimates. British Journal of Educational Psychology, 90(3), 683-699. https://doi.org/10.1111/bjep.12325

Furnham, A., \& Bachtiar, V. (2008). Personality and intelligence as predictors of creativity. Personality and Individual Differences, 45(7), 613-617. https://doi.org/10.1016/j.paid.2008.06.023

Gibson, C., Folley, B. S., \& Park, S. (2009). Enhanced divergent thinking and creativity in musicians: A behavioral and near-infrared spectroscopy study. Brain and Cognition, 69(1), 162-169. https://doi.org/10.1016/j.bandc.2008.07.009

Gilhooly, K. J., Fioratou, E., Anthony, S. H., \& Wynn, V. (2007). Divergent thinking: Strategies and executive involvement in generating novel uses for familiar 
objects. British Journal of Psychology, 98(4), 611-625.

https://doi.org/10.1111/j.2044-8295.2007.tb00467.x

Gilhooly, K. J., Georgiou, G., \& Devery, U. (2013). Incubation and creativity: Do something different. Thinking \& Reasoning, 19(2), 137-149. https://doi.org/10.1080/13546783.2012.749812

Gilhooly, Kenneth J., Georgiou, G. J., Garrison, J., Reston, J. D., \& Sirota, M. (2012). Don't wait to incubate: Immediate versus delayed incubation in divergent thinking. Memory \& Cognition, 40(6), 966-975. https://doi.org/10.3758/s13421012-0199-z

Gocłowska, M. A., Ritter, S. M., Elliot, A. J., \& Baas, M. (2019). Novelty seeking is linked to openness and extraversion, and can lead to greater creative performance. Journal of Personality, 87(2), 252-266. https://doi.org/10.1111/jopy.12387

Guilford, J. P. (1967). Creativity: Yesterday, today and tomorrow. The Journal of Creative Behavior, 1(1), 3-14. https://doi.org/10.1002/j.21626057.1967.tb00002.x

Han, K.-S. (2003). Domain-Specificity of Creativity in Young Children: How Quantitative and Qualitative Data Support It. The Journal of Creative Behavior, 37(2), 117-142. https://doi.org/10.1002/j.2162-6057.2003.tb00829.x

Hass, R. W. (2015). Feasibility of online divergent thinking assessment. Computers in Human Behavior, 46, 85-93. https://doi.org/10.1016/j.chb.2014.12.056

Hocevar, D. (1979). Ideational fluency as a confounding factor in the measurement of originality. Journal of Educational Psychology, 71(2), 191-196. https://doi.org/10.1037/0022-0663.71.2.191 
Hocevar, D., \& Michael, W. B. (1979). The Effects of Scoring Formulas on the Discriminant Validity of Tests of Divergent Thinking. Educational and Psychological Measurement, 39(4), 917-921. https://doi.org/10.1177/001316447903900427

Johns, G. A., \& Morse, L. W. (1997). Divergent thinking as a function of time and prompting to "be creative" in undergraduates. The Journal of Creative Behavior, 31(2), 156-165. https://doi.org/10.1002/j.2162-6057.1997.tb00788.x

Jones, L. L., \& Estes, Z. (2015). Convergent and divergent thinking in verbal analogy. Thinking \& Reasoning, 21(4), 473-500. https://doi.org/10.1080/13546783.2015.1036120

Kaufman, J. C., \& Beghetto, R. A. (2009). Beyond Big and Little: The Four C Model of Creativity. Review of General Psychology, 13(1), 1-12. https://doi.org/10.1037/a0013688

Kilgour, M. (2006). Improving the creative process: Analysis of the effects of divergent thinking techniques and domain specific knowledge on creativity. International Journal of Business and Society, 7(2), 79-107.

Kim, K. H. (2008). Meta-analyses of the relationship of creative achievement to both IQ and divergent thinking test scores. The Journal of Creative Behavior, 43, 106130. https://doi.org/10.1002/j.2162-6057.2008.tb01290.x

Koo, T. K., \& Li, M. Y. (2016). A Guideline of Selecting and Reporting Intraclass Correlation Coefficients for Reliability Research. Journal of Chiropractic Medicine, 15(2), 155-163. https://doi.org/10.1016/j.jcm.2016.02.012.

McClain, M.-C., \& Pfeiffer, S. (2012). Identification of gifted students in the united states 
today: A look at state definitions, policies, and practices. Journal of Applied School Psychology, 28(1), 59-88. https://doi.org/10.1080/15377903.2012.643757 McCrae, R. R. (1987). Creativity, Divergent Thinking, and Openness to Experience. Journal of Personality and Social Psychology, 52(6), 1258-1265.

McGraw, K. O., \& Wong, S. P. (1996). Forming inferences about some intraclass correlation coefficients. Psychological Methods, 1(1), 30-46. https://doi.org/10.1037/1082-989X.1.1.30

Nicholson, M. W., \& Moran, J. D. (1986). Teachers' judgments of preschoolers' creativity. Perceptual and Motor Skills, 63(3), 1211-1216. https://doi.org/10.2466/pms.1986.63.3.1211

Nusbaum, E. C., \& Silvia, P. J. (2011). Are intelligence and creativity really so different? Fluid intelligence, executive processes, and strategy use in divergent thinking. Intelligence, 39(1), 36-45. https://doi.org/10.1016/j.intell.2010.11.002

Paletz, S. B. F., \& Schunn, C. D. (2010). A social-cognitive framework of multidisciplinary team innovation. Topics in Cognitive Science, 2(1), 73-95. https://doi.org/10.1111/j.1756-8765.2009.01029.x

Pang, W. (2015). Promoting creativity in the classroom: A generative view. Psychology of Aesthetics, Creativity, and the Arts, 9(2), 122-127. https://doi.org/10.1037/aca0000009

Park, N. K., Chun, M. Y., \& Lee, J. (2016). Revisiting individual creativity assessment: Triangulation in subjective and objective assessment methods. Creativity Research Journal, 28(1), 1-10. https://doi.org/10.1080/10400419.2016.1125259

Plucker, J. A., Beghetto, R. A., \& Dow, G. T. (2004). Why Isn't Creativity More 
Important to Educational Psychologists? Potentials, Pitfalls, and Future Directions in Creativity Research. Educational Psychologist, 39(2), 83-96. https://doi.org/10.1207/s15326985ep3902_1

Plucker, J. A., Qian, M., \& Wang, S. (2011). Is originality in the eye of the beholder? Comparison of scoring techniques in the assessment of divergent thinking. The Journal of Creative Behavior, 45(1), 1-22. https://doi.org/10.1002/j.21626057.2011.tb01081.x

Rammstedt, B., \& John, O. P. (2007). Measuring personality in one minute or less: A 10item short version of the Big Five Inventory in English and German. Journal of Research in Personality, 41(1), 203-212. https://doi.org/10.1016/j.jrp.2006.02.001

Reynolds, C. R., \& Kamphaus, R. W. (2005). Introduction to the Reynolds Intellectual Assessment Scales and the Reynolds Intellectual Screening Test. In Contemporary Intellectual Assessment: Theories, Tests, and Issues. (pp. 461483). The Guilford Press.

Roberts, P. (2006). Nurturing Creativity in Young People: A report to Government to inform future policy. Department for culture, media and sport.

Runco, M. A. (1986). Flexibility and Originality in Children's Divergent Thinking. The Journal of Psychology, 120(4), 345-352.

https://doi.org/10.1080/00223980.1986.9712632

Runco, M. A., Abdulla, A. M., Paek, S. H., Al-Jasim, F. A., \& Alsuwaidi, H. N. (2016). Which Test of Divergent Thinking Is Best? Creativity. Theories - Research Applications, 3(1), 4-18. https://doi.org/10.1515/ctra-2016-0001 
Runco, M. A., \& Acar, S. (2012). Divergent thinking as an indicator of creative potential. Creativity Research Journal, 24(1), 1-10. https://doi.org/10.1080/10400419.2012.652929

Runco, M. A., Okuda, S. M., \& Thurston, B. J. (1987). The Psychometric Properties of Four Systems for Scoring Divergent Thinking Tests: Journal of Psychoeducational Assessment. https://doi.org/10.1177/073428298700500206

Shrout, P. E., \& Fleiss, J. L. (1979). Intraclass Correlations: Uses in Assessing Rater Reliability. Psychological Bulletin, 86(2), 420-428.

Silvia, P. J., Martin, C., \& Nusbaum, E. C. (2009). A snapshot of creativity: Evaluating a quick and simple method for assessing divergent thinking. Thinking Skills and Creativity, 4(2), 79-85. https://doi.org/10.1016/j.tsc.2009.06.005

Silvia, P. J., Winterstein, B. P., \& Willse, J. T. (2008a). Rejoinder: The madness to our method: Some thoughts on divergent thinking. Psychology of Aesthetics, Creativity, and the Arts, 2(2), 109-114. https://doi.org/10.1037/19313896.2.2.109

Silvia, P. J., Winterstein, B. P., Willse, J. T., Barona, C. M., Cram, J. T., Hess, K. I., Martinez, J. L., \& Richard, C. A. (2008b). Assessing creativity with divergent thinking tasks: Exploring the reliability and validity of new subjective scoring methods. Psychology of Aesthetics, Creativity, and the Arts, 2(2), 68-85. https://doi.org/10.1037/1931-3896.2.2.68

Sio, U. N., \& Ormerod, T. C. (2009). Does incubation enhance problem solving? A metaanalytic review. Psychological Bulletin, 135(1), 94-120. https://doi.org/10.1037/a0014212 
Smith, S. M., \& Blankenship, S. E. (1991). Incubation and the Persistence of Fixation in Problem Solving. The American Journal of Psychology, 104(1), 61. https://doi.org/10.2307/1422851

Torrance, E. P. (1966). Torrance tests of creative thinking: Norms-technical manual: Verbal tests, forms $a$ and b: Figural tests, forms $a$ and $b$. Personal Press, Incorporated.

Wallach, M. A., \& Kogan, N. (1965). Modes of thinking in young children. New York. Wallas, G. (1926). The art of thought. New York : Harcourt, Brace and Co.

Weiss, S., Steger, D., Kaur, Y., Hildebrandt, A., Schroeders, U., \& Wilhelm, O. (2020). On the Trail of Creativity: Dimensionality of Divergent Thinking and its Relation with Cognitive Abilities, Personality, and Insight. European Journal of Personality. https://doi.org/10.1002/per.2288

Wilson, R. C., Guilford, J. P., \& Christensen, P. R. (1953). The measurement of individual differences in originality. Psychological Bulletin, 50(5), 362-370. https://doi.org/10.1037/h0060857 


\section{Table 1}

Descriptive Statistics for All Primary Study Variables

\begin{tabular}{|c|c|c|c|c|}
\hline Variable & $N$ & Range & M or $\%$ & $S D$ \\
\hline \multicolumn{5}{|l|}{ Child Demographic Information } \\
\hline Gender $($ Female $=1)$ & 42 & 0,1 & $64 \%$ & - \\
\hline Race/Ethnicity (White $=1$ ) & 42 & 0,1 & $79 \%$ & - \\
\hline Age in Years & 42 & $3.05-6.98$ & 4.84 & 1.21 \\
\hline \multicolumn{5}{|l|}{ Child Originality Scores - Instances } \\
\hline Objective & 42 & $0-1.17$ & .57 & .34 \\
\hline Subjective & 42 & $1-3.04$ & 3.80 & 1.06 \\
\hline Personal - Short Incubation & 42 & $0-13$ & 4.69 & 3.43 \\
\hline Personal - Long Incubation & 40 & $0-16$ & 5.43 & 3.10 \\
\hline Fluency & 42 & $1-15$ & 6.95 & 3.33 \\
\hline \multicolumn{5}{|c|}{ Child Originality Scores - Alternative Uses } \\
\hline Objective & 42 & $0-1.17$ & .44 & .35 \\
\hline Subjective & 42 & $1-3.04$ & 1.70 & .54 \\
\hline Personal - Short Incubation & 42 & $0-9$ & 2.64 & 2.22 \\
\hline Personal - Long Incubation & 40 & $0-8$ & 2.10 & 1.84 \\
\hline Fluency & 42 & $1-14$ & 5.1 & 3.09 \\
\hline \multicolumn{5}{|l|}{ Parent Ratings of Children } \\
\hline Openness to Experience & 42 & $4-10$ & 6.76 & 1.32 \\
\hline Extraversion & 42 & $3-6$ & 5.05 & .94 \\
\hline Creativity & 42 & $3-10$ & 7.00 & 1.75 \\
\hline \multicolumn{5}{|l|}{ Child Performance Measures } \\
\hline Defined Creativity $(\mathrm{Yes}=1)$ & 42 & 0,1 & $43 \%$ & - \\
\hline Creative Drawing & 42 & $1-5$ & 2.60 & 1.23 \\
\hline Creative Title & 42 & $1-5$ & 2.74 & 1.38 \\
\hline Combined Drawing/Title & 42 & $2.5-10$ & 5.37 & 2.25 \\
\hline Verbal Reasoning & 42 & $0-5$ & 3.24 & 1.62 \\
\hline
\end{tabular}

Note. Subjective, Creative Drawing, and Creative Title scores represent the average score of two raters. Combined Drawing/Title scores represent the sum of Creative Drawing and Creative Title scores. 


\section{Table 2}

Intraclass Correlation Coefficients for Subjective Ratings, Drawings, and Drawing Titles

\begin{tabular}{llcccc}
\hline & ICC $(95 \%$ & \multicolumn{3}{c}{ F Test with True Value 0 } \\
\cline { 3 - 6 } Measure & Confidence Interval) & Value & $d f 1$ & $d f 2$ & $\begin{array}{l}\text { p- } \\
\text { Value }\end{array}$ \\
\hline $\begin{array}{l}\text { Creative } \\
\text { Drawings }\end{array}$ & $0.740(0.508-0.861)$ & 4.180 & 41 & 41 & .000 \\
$\begin{array}{l}\text { Creative } \\
\text { Titles }\end{array}$ & $0.775(0.577-0.879)$ & 4.744 & 41 & 41 & .000 \\
$\begin{array}{l}\text { Subjective } \\
\text { IT }\end{array}$ & $0.747(0.623-0.825)$ & 4.386 & 179 & 179 & .000 \\
$\begin{array}{l}\text { Subjective } \\
\text { AUT }\end{array}$ & $0.813(0.685-0.882)$ & 6.207 & 153 & 153 & .000 \\
\hline
\end{tabular}

Note. Average measures, two-way mixed effects models with absolute agreement definition. $\mathrm{IT}=$ Instances Task. $\mathrm{AU}=$ Alternative Uses Task.

$* p<.05 . * * p<.01 . * * * p<.001$. 


\section{Table 3}

Correlations Among Instances Task Scores and Other Primary Study Variables

\begin{tabular}{|c|c|c|c|c|c|c|c|c|c|c|c|c|c|c|}
\hline & $N$ & 1. & 2. & 3. & 4. & 5. & 6. & 7. & 8. & 9. & 10. & 11. & 12. & 13. \\
\hline 1. Age in Years & 42 & & & & & & & & & & & & & \\
\hline 2. Objective IT & 42 & .072 & & & & & & & & & & & & \\
\hline 3. Subjective IT & 42 & .034 & .181 & & & & & & & & & & & \\
\hline 4. Short PO IT & 42 & $.514 * *$ & .234 & .006 & & & & & & & & & & \\
\hline 5. Long PO IT & 40 & .189 & $.317 *$ & .051 & $.327 *$ & & & & & & & & & \\
\hline 6. Fluency IT & 42 & $.386^{*}$ & .247 & .055 & $.338^{*}$ & $.494 * *$ & & & & & & & & \\
\hline $\begin{array}{l}\text { 7. Openness to } \\
\text { Experiences }\end{array}$ & 42 & .061 & .189 & .073 & -.178 & .088 & .258 & & & & & & & \\
\hline 8. Extraversion & 42 & -.092 & -.095 & .010 & -.238 & -.091 & -.007 & -.168 & & & & & & \\
\hline 9. Parent Rating CC & 42 & .226 & .279 & -.086 & $.454 * *$ & .131 & .226 & -.042 & -.119 & & & & & \\
\hline $\begin{array}{l}\text { 10. Defined } \\
\text { Creativity }(\text { Yes }=1)\end{array}$ & 42 & $.433 * *$ & .132 & .157 & $.306^{*}$ & .095 & .100 & -.174 & -.045 & -.028 & & & & \\
\hline $\begin{array}{l}\text { 11. Creative } \\
\text { Drawing }\end{array}$ & 42 & $.515^{* * *}$ & .219 & .129 & $.564 * * *$ & $.399 *$ & $.518 * * *$ & -.091 & .017 & $.323 *$ & $.309 *$ & & & \\
\hline 12. Creative Title & 42 & $.309 *$ & .133 & .145 & $.380 *$ & $.464 * *$ & $.347 *$ & .045 & 0.057 & .126 & .254 & $.565 * * *$ & & \\
\hline $\begin{array}{l}\text { 13. Combined } \\
\text { Drawing/Title }\end{array}$ & 42 & $.462 * *$ & .189 & .159 & $.521 * * *$ & $.482 * *$ & $.518 * * *$ & -.039 & 0.061 & .253 & $.364 *$ & $.872 * * *$ & $.882 * * *$ & \\
\hline $\begin{array}{l}\text { 14. Verbal } \\
\text { Reasoning }\end{array}$ & 42 & $.610^{* * *}$ & .138 & .004 & $.479 * *$ & $.321 *$ & $.455^{* *}$ & .004 & -.104 & .275 & $.442 * *$ & $.473 * *$ & $.448 * *$ & $.504 * *$ \\
\hline
\end{tabular}

Note. $\mathrm{IT}=$ Instances Task. $\mathrm{PO}=$ Personal Originality. $\mathrm{CC}=$ Child Creativity.

$* p<.05 . * * p<.01 . * * * p<.001$. 


\section{Table 4}

Correlations Among Alternative Uses Task Scores and Other Primary Study Variables

\begin{tabular}{|c|c|c|c|c|c|c|c|c|c|c|c|c|c|c|}
\hline & $N$ & 1. & 2. & 3. & 4. & 5. & 6. & 7. & 8. & 9. & 10. & 11. & 12. & 13. \\
\hline 1. Age in Years & 42 & & & & & & & & & & & & & \\
\hline 2. Objective AUT & 42 & .201 & & & & & & & & & & & & \\
\hline 3. Subjective AUT & 42 & $.402 * *$ & $.428 * *$ & & & & & & & & & & & \\
\hline 4. Short PO AUT & 42 & $.524 * * *$ & .300 & $.565 * * *$ & & & & & & & & & & \\
\hline 5. Long PO AUT & 40 & -.049 & -.056 & .184 & .195 & & & & & & & & & \\
\hline 6. Fluency AUT & 42 & $.322 *$ & .223 & $.556 * * *$ & $.429 * *$ & $.356^{*}$ & & & & & & & & \\
\hline $\begin{array}{l}\text { 7. Openness to } \\
\text { Experiences }\end{array}$ & 42 & .061 & .092 & .209 & .112 & .094 & .179 & & & & & & & \\
\hline 8. Extraversion & 42 & -.092 & -.171 & -.112 & -.227 & -.090 & -.010 & -.168 & & & & & & \\
\hline $\begin{array}{l}\text { 9. Parent Rating CC } \\
\text { 10. Defined }\end{array}$ & 42 & .226 & .302 & .105 & $.320 *$ & .029 & .041 & -.042 & -.119 & & & & & \\
\hline Creativity $($ Yes $=1)$ & 42 & $.433 * *$ & -.125 & .207 & $.317 *$ & .036 & .272 & -.174 & -.045 & -.028 & & & & \\
\hline $\begin{array}{l}\text { 11. Creative } \\
\text { Drawing }\end{array}$ & 42 & $.515 * * *$ & .156 & $.391 *$ & $.425 * *$ & .096 & .220 & -.091 & .017 & $.323 *$ & $.309 *$ & & & \\
\hline 12. Creative Title & 42 & $.309 *$ & .137 & $.417 * *$ & $.314 *$ & .119 & .177 & .045 & 0.057 & .126 & .254 & $.565 * * *$ & & \\
\hline $\begin{array}{l}\text { 13. Combined } \\
\text { Drawing/Title }\end{array}$ & 42 & $.462 * *$ & .152 & $.439 * *$ & $.403 * *$ & .116 & .221 & -.039 & 0.061 & .253 & $.364 *$ & $.872 * * *$ & $.882 * * *$ & \\
\hline $\begin{array}{l}\text { 14. Verbal } \\
\text { Reasoning }\end{array}$ & 42 & $.610 * * *$ & .165 & $.370^{*}$ & $.499 * *$ & .188 & $.366^{*}$ & .004 & -.104 & .275 & $.442 * *$ & $.473 * *$ & $.448 * *$ & $.504 * *$ \\
\hline
\end{tabular}

Note . $\mathrm{PO}=$ Personal Originality. AUT $=$ Alternative Uses Task. $\mathrm{CC}=$ Child Creativity.

$* p<.05 . * * p<.01 . * * * p<.001$. 


\section{Table 5}

Correlations Among Divergent Thinking Task Outcomes

\begin{tabular}{|c|c|c|c|c|c|c|c|c|c|c|}
\hline & $N$ & 1 & 2 & 3 & 4 & 5 & 6 & 7 & 8 & 9 \\
\hline 1. Objective AUT & 42 & & & & & & & & & \\
\hline 2. Subjective AUT & 42 & $.428 * *$ & & & & & & & & \\
\hline 3. Short PO AUT & 42 & .300 & $.565 * * *$ & & & & & & & \\
\hline 4. Long PO AUT & 40 & -.056 & .184 & .195 & & & & & & \\
\hline 5. Fluency AUT & 42 & .223 & $.556 * * *$ & $.429 * *$ & $.356^{*}$ & & & & & \\
\hline 6. Objective IT & 42 & -.092 & $.399 * *$ & .239 & $.375^{*}$ & .303 & & & & \\
\hline 7. Subjective IT & 42 & .124 & $.376^{*}$ & .162 & .122 & $.358^{*}$ & .181 & & & \\
\hline 8. Short PO IT & 42 & .090 & .259 & $.527 * * *$ & .210 & .148 & .234 & .006 & & \\
\hline 9. Long PO IT & 40 & -.043 & $.388 *$ & .243 & $.622 * * *$ & .278 & $.317 *$ & .051 & $.327 *$ & \\
\hline 10. Fluency IT & 42 & .190 & $.448 * *$ & $.378 *$ & .301 & $.506 * *$ & .247 & .055 & $.338 *$ & $.494 * *$ \\
\hline
\end{tabular}

Note. AUT $=$ Alternative Uses Task. $\mathrm{PO}=$ Personal Originality. $\mathrm{IT}=$ Instances Task.

$* p<.05 . * * p<.01 . * * * p<.001$. 


\section{Table 6}

Results of Linear Regression Analyses Examining the Influence of Child Age and Task-Specific Fluency on Personal Originality

\begin{tabular}{cccccc}
\hline DV & Predictors & $B(S E)$ & $\beta$ & $t$ & $p$ \\
\hline $\begin{array}{c}\text { AUT Short } \\
\text { PO }\end{array}$ & Age & $.76(.14)$ & .50 & 5.31 & $<.001$ \\
& Fluency & $.32(.07)$ & .53 & 5.63 & $<.001$ \\
IT Short & Age & $1.46(.39)$ & .51 & 3.79 & $<.05$ \\
PO & & & & \\
IT Long & Age & $-.03(.40)$ & -.01 & -.08 & $N S$ \\
PO & Fluency & $.48(.15)$ & .50 & 3.19 & $<.05$ \\
& & & & & \\
\hline
\end{tabular}

Note. AUT $=$ Alternative Uses Task. $\mathrm{PO}=$ Personal Originality. $\mathrm{IT}=$ Instances Task. $N S=$ not statistically significant. 


\section{Table 7}

Hierarchical Linear Regression Exploring the Effects of Age and Personal-Originality Scores on Children's Combined Drawing/Title Scores

\begin{tabular}{llcccccccc}
\hline Step & Predictors & $B$ & $S E$ & $t$ & $p$ & $R^{2}$ & $\begin{array}{c}R^{2} \\
\text { Change }\end{array}$ & $\begin{array}{c}F \\
\text { Change }\end{array}$ & $p$ \\
\hline 1 & Age & .86 & .26 & 3.30 & .002 & .21 & .21 & 10.86 & .002 \\
2 & Age & .65 & .30 & 2.12 & .040 & .25 & .04 & 1.85 & .182 \\
& AUT Short PO & .23 & .17 & 1.36 & .182 & & & & \\
1 & Age & .86 & .26 & 3.30 & .002 & .21 & .21 & 10.86 & .002 \\
2 & Age & .49 & .29 & 1.72 & .094 & .32 & .11 & 6.32 & .016 \\
& IT Short PO & .25 & .10 & 2.51 & .016 & & & & \\
1 & Age & .86 & .26 & 3.36 & .002 & .23 & .23 & 11.31 & .002 \\
2 & Age & .75 & .25 & 3.07 & .004 & .39 & .16 & 9.61 & .004 \\
& IT Long PO & .29 & .10 & 3.1 & .004 & & & & \\
\hline
\end{tabular}

Note . AUT $=$ Alternative Uses Task. $\mathrm{IT}=$ Instances Task. $\mathrm{PO}=$ Personal Originality. 


\section{Figure 1}

Sample Drawing with Title for Performance Measure of Creativity

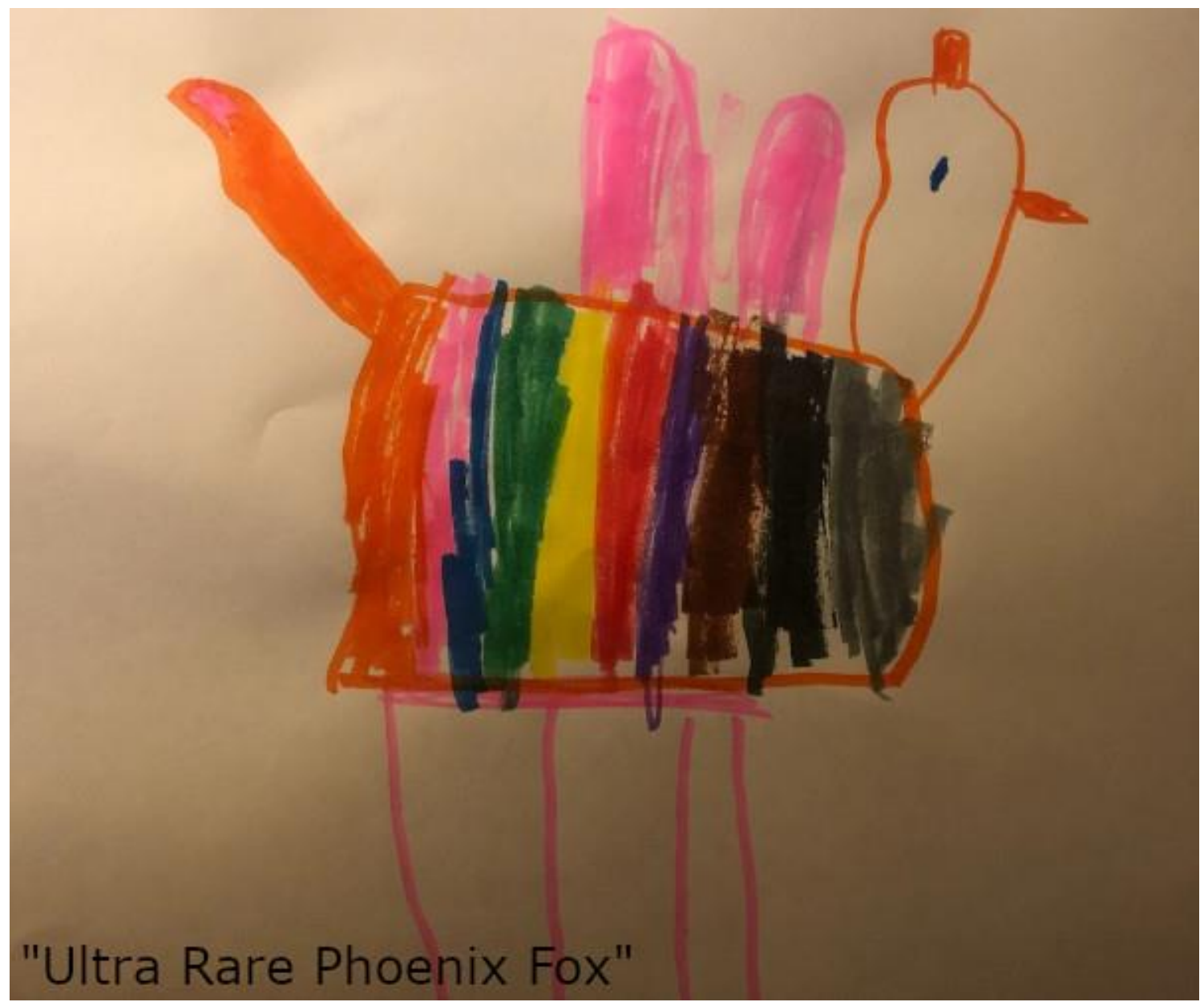

Note. Titles were not visible during rating of drawing. This child received a " 5 " rating for drawing and title by both raters. 
Figure 2

Screenshot of Zoom Session Screen-Share During Instances Task
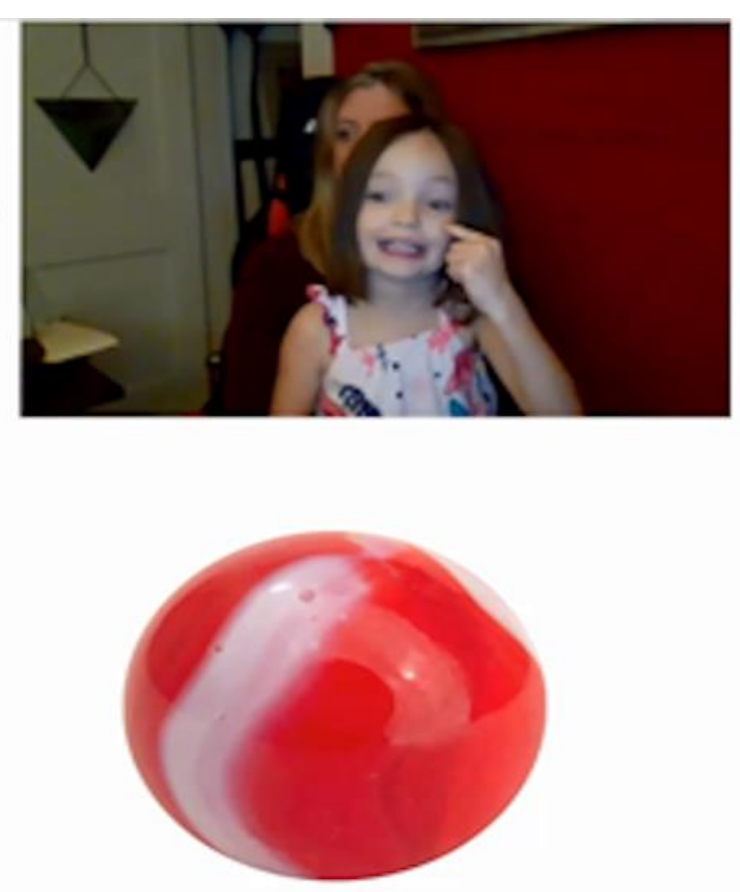

Note. Image reflects experimenter's point of view. 


\section{Figure 3}

Screenshot of Zoom Session Screen-Share During Alternative Uses Task

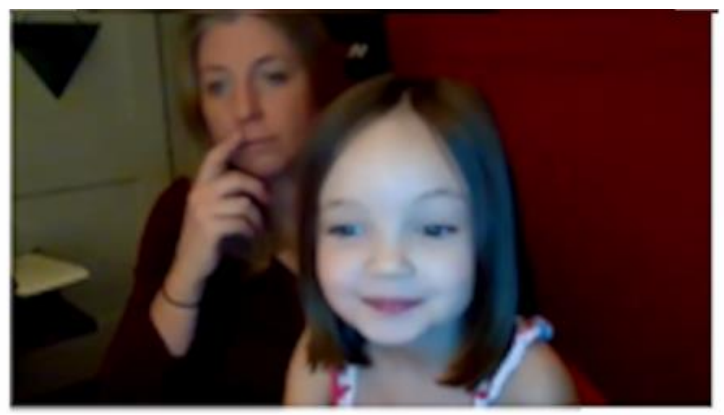

Note. Image reflects experimenter's point of view. 


\section{Figure 4}

Coding Raw Responses for Objective Originality Scoring

Raw Responses

Color

Color a spider

Coloring

Coloring eyes

Draw

Draw a face

Drawing

Drawing cats

Drawing a house

Drawing on a wall
Categorically Identical Responses

Color

Coloring

Color a spider

Coloring eyes

Draw

Drawing

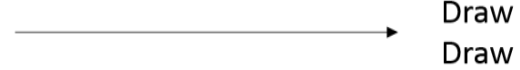

Draw a face

Drawing cats

Drawing a house

Drawing on a wall

\section{Coded Responses}

Color

Color

Color specific

Color specific

Draw

Draw specific

Draw specific

Draw specific

Drawing On

Note. Responses sampled from alternative uses task ideational pool. 
Figure 5

Developmental Trajectories of Instances Task Fluency and Originality Scores

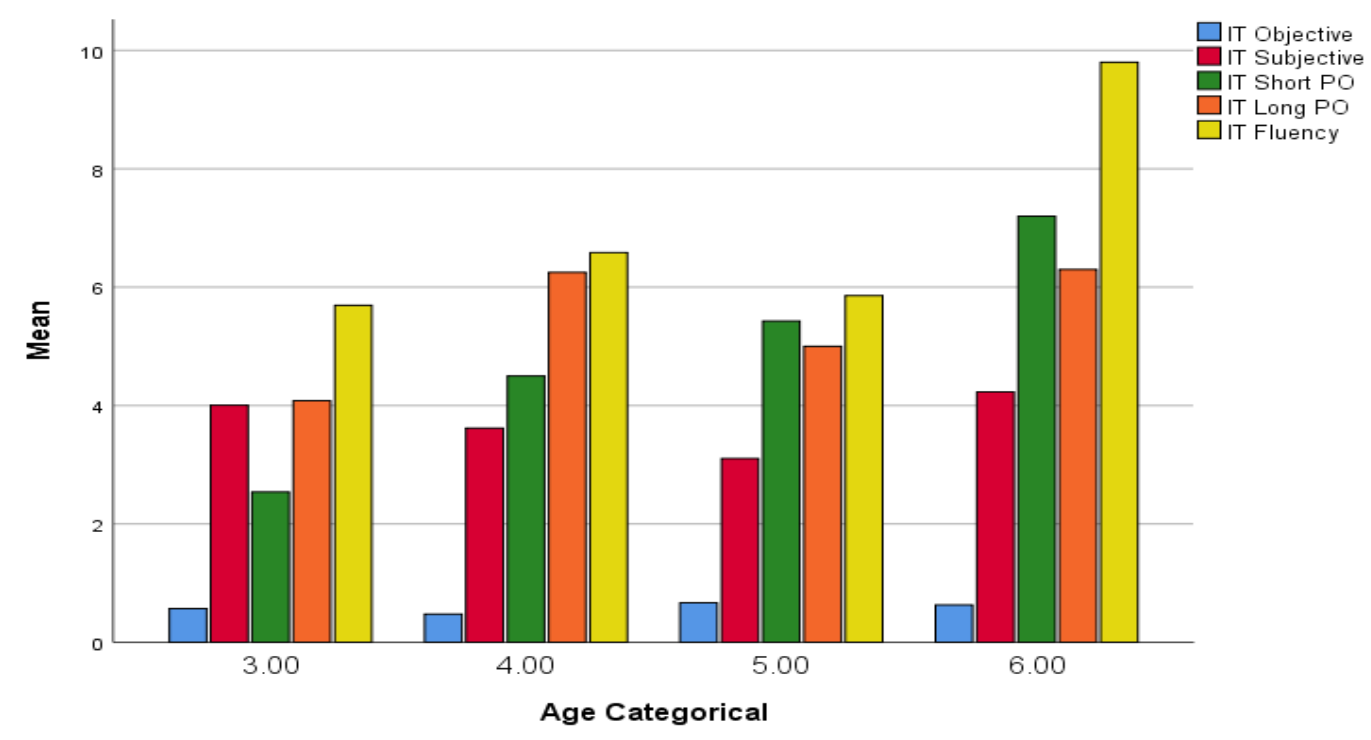

Note. IT $=$ Instances Task. $\mathrm{PO}=$ Personal Originality. 


\section{Figure 6}

Developmental Trajectories of Alternative Uses Task Fluency and Originality Scores

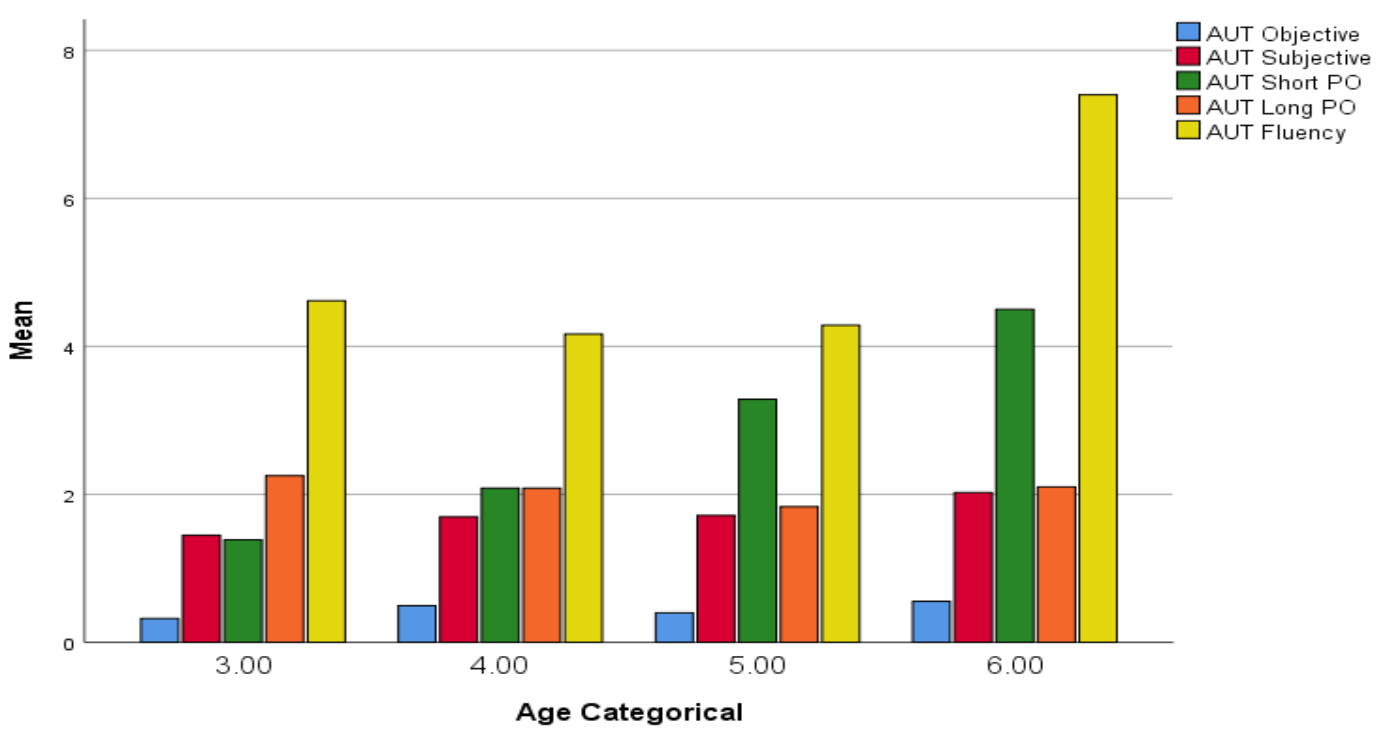

Note . AUT $=$ Alternative Uses Task. $\mathrm{PO}=$ Personal Originality. 


\section{Figure 7}

Visual Representation of Within-Session Personal Originality Mediating the Relation Between Age and Combined Drawing/Title Creativity Ratings
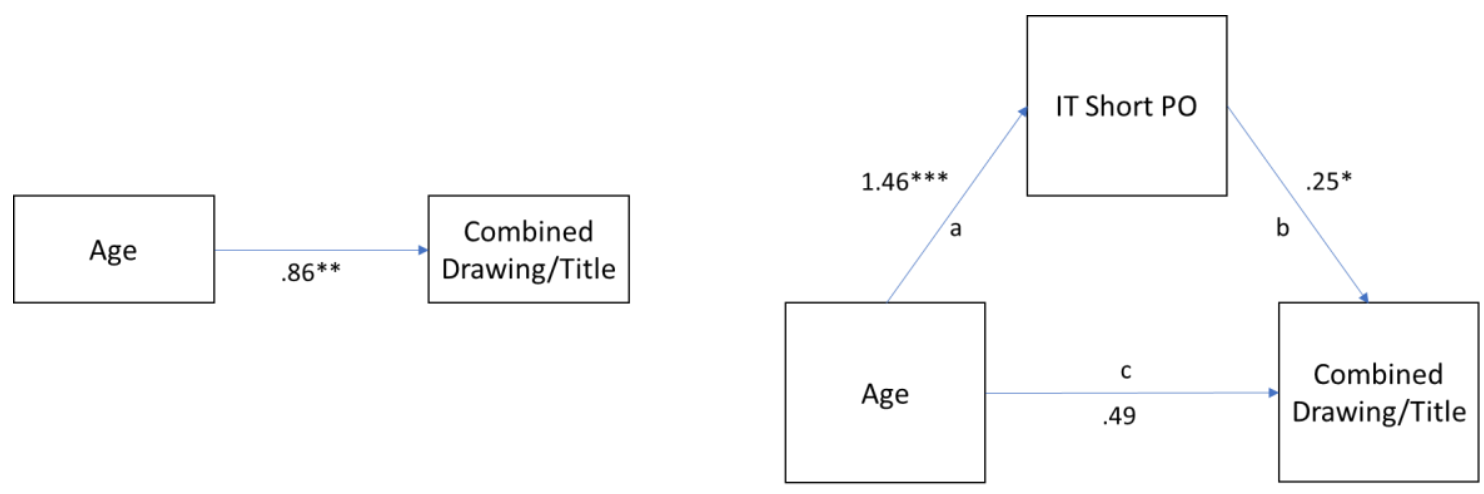

Indirect Effect $(\mathrm{a} * \mathrm{~b})=.37(.18), p<.05$

Mean bootstrapped effect $=.36(.18), 95 \% \mathrm{Cl} .052$ to .777

Note IT $=$ Instances Task. $\mathrm{PO}=$ Personal Originality.

$* p<.05 . * * p<.01 . * * * p<.001$. 


\section{Appendix A}

\section{Originality Scoring Protocol}

This appendix depicts the step-by-step process through which divergent thinking task responses were organized and scored. Objective, Subjective, Short PersonalOriginality, and Long Personal-Originality scoring protocols are presented in two sections: preparation and scoring.

\section{Objective Originality Scoring Protocol}

\section{Preparation}

1. Two pre-incubation ideational pools were generated: one for instances and one for alternative uses.

2. Responses were organized in alphabetical order and by Child ID.

3. Identical, near identical, and categorically identical responses were collapsed to ensure fair and accurate originality scoring (see Figure 4 for examples).

4. Excel-formulas were used to detect responses at two levels of statistical rarity: $10 \%$ and $5 \%$.

\section{Scoring}

1. Responses produced by $<10 \%$ of the sample received a score of 1 ; responses produced by $<5 \%$ of the sample received a score of 2 .

2. Ratio scores for instances and alternative uses were calculated by dividing the sum of weighted scores by the total number of responses.

\section{Subjective Originality Scoring Protocol}

\section{Preparation}

1. Two pre-incubation ideational pools were generated: one for instances and one for alternative uses.

2. Responses were organized in alphabetical order and de-identified.

3. Raters conducted a thorough inspection of the entire (alphabetized) ideational pool.

4. After inspection, raters received an abridged ideational pool such that each

Scoring response was only listed once.

1. Raters assigned a score of 1 (not at all creative) to 5 (highly creative) to all responses in the abridged pool based on: uncommonness, remoteness, and cleverness (see Appendix C).

2. The average of ratings for each response were repopulated to the full ideational pool with child identifiers.

3. Ratio scores for instances and alternative uses were calculated by dividing the sum of subjective scores by the total number of responses. 


\section{Short Personal-Originality Scoring Protocol}

\section{Preparation}

1. Complete ideational pools were generated by child ID and separated into two columns: pre-filler task and post-filler task.

2. Excel formulas were used to detect new responses (within-child) in the postincubation column.

\section{Scoring}

1. Short personal-originality scores for instances and alternative uses were measured as the total number of new responses in post-filler task lists.

\section{Long Personal-Originality Scoring Protocol}

\section{Preparation}

1. Complete ideational pools were generated by child ID and separated into two columns: all Time 1 responses (i.e., pre- and post-filler task) and all Time 2 responses (collected in part 2 of the parent survey).

2. Excel formulas were used to detect new responses (within-child) in the Time 2 responses column.

\section{Scoring}

1. Long personal-originality scores for instances and alternative uses were measured as the total number of new responses in the Time 2 response list. 


\section{Appendix B}

\section{Measures and Child Task Instructions}

This appendix presents all primary study measures not fully described in the manuscript, including the personality measure from the parent survey and tasks completed during child zoom sessions.

\section{Big Five Inventory 10 - Child Form (BFI-10)}

The BFI-10 was used to measure children's openness to experience (the sum of item 5 reverse coded and item 10) and extraversion (the sum of item 1 reverse coded and item 6).

\section{Instructions}

Here are a number of characteristics that may or may not apply to your child. For example, do you agree that your child is someone who likes to spend time with others? Please circle the number to indicate the extent to which you agree or disagree with that statement.

\begin{tabular}{|c|c|c|c|c|c|c|}
\hline \multicolumn{2}{|c|}{ I see my child as someone who... } & \multirow{2}{*}{$\begin{array}{c}\begin{array}{c}\text { Disagree } \\
\text { Strongly }\end{array} \\
1\end{array}$} & \multirow{2}{*}{$\begin{array}{l}\begin{array}{l}\text { Disagree } \\
\text { a Little }\end{array} \\
2\end{array}$} & \multirow{2}{*}{$\begin{array}{l}\text { Neither } \\
\text { Agree nor } \\
\text { Disagree } \\
3\end{array}$} & \multirow{2}{*}{$\begin{array}{l}\begin{array}{l}\text { Agree A } \\
\text { little }\end{array} \\
4\end{array}$} & \multirow{2}{*}{$\begin{array}{r}\begin{array}{l}\text { Agree } \\
\text { Strongly }\end{array} \\
5\end{array}$} \\
\hline 1. & $\ldots$ is reserved & & & & & \\
\hline 2. & ...is generally trusting & 1 & 2 & 3 & 4 & 5 \\
\hline 3. & ...tends to be lazy & 1 & 2 & 3 & 4 & 5 \\
\hline 4. & $\begin{array}{l}\text {...is relaxed, handles stress } \\
\text { well }\end{array}$ & 1 & 2 & 3 & 4 & 5 \\
\hline 5. & ...has few artistic interests & 1 & 2 & 3 & 4 & 5 \\
\hline 6. & ...is outgoing, sociable & 1 & 2 & 3 & 4 & 5 \\
\hline 7. & $\begin{array}{l}\text {...tends to find fault with } \\
\text { others }\end{array}$ & 1 & 2 & 3 & 4 & 5 \\
\hline 8. & ...does a thorough job & 1 & 2 & 3 & 4 & 5 \\
\hline 9. & ...gets nervous easily & 1 & 2 & 3 & 4 & 5 \\
\hline 10. & ...has an active imagination & 1 & 2 & 3 & 4 & 5 \\
\hline
\end{tabular}

\section{Child Task Instructions}

Below is the exact script used during the child zoom sessions detailing specific instructions for each child task. Note the order of child tasks was randomized with the 
exception of define creativity, which occurred first in each session, and verbal reasoning which occurred last in each session.

\section{Define Creativity}

1.1. Okay \{child name $\}$ have you ever heard the word "creative" before? I was wondering if you could tell me what the word creative means?

$>$ If child is unsure, try two follow up prompts $\rightarrow$ Can you give me your best guess for what the word creative means? How about you give it your best shot?

$>$ If unsure/unresponsive/inaccurate, transition to 1.2. If participant demonstrates an understanding of the word creative, transition to 1.3

1.2. That's okay, \{child name $\}$ When someone is creative, it means they come up with new and interesting ideas. We are going to play a few games where I want you to show me how creative you can be. Being creative is fun because there is never a wrong answer!

1.3. That's right, \{child name\} [reaffirm participant's definition here]. When someone is creative, it means they come up with new and interesting ideas. We are going to play a few games where I want you to show me how creative you can be. Being creative is fun because there is never a wrong answer!

\section{Alternative Uses Task}

2.1. [Display pencil on screen and hold pencil to camera]. Do you know what this is?

$>$ If unsure/unresponsive/inaccurate: This is a pencil

$>$ If accurate: That's right! [reaffirm child's response].

2.2. Can you tell me what a pencil is used for?

$>$ If unsure/unresponsive/inaccurate: Sometimes we use a pencil for things like writing our name! [demonstrate]

$>$ If accurate: That's right! [reaffirm child's response].

2.3. In this game, I want you to tell me all of the ways you could use a pencil! Try to think of all the ways you could use a pencil and be as creative as you can.

$>$ Positively reaffirm every response.

$>$ In the case of $a>5$ second pause $\rightarrow$ Can you think of any more ways you could use a pencil?

$>$ Once participant communicates s/he can no longer think of more responses, transition to filler task. After filler task, transition to 2.4

2.4. Nice work, \{child name $\}$. Do you remember the game we were playing about things we could use a pencil for? You came up with a lot of creative things you could use a pencil for. You said you could use a pencil for [list participant's responses here]. Can you think of any more creative ways you could use a pencil?

$>$ Positively reaffirm every response.

$>$ In the case of $a>5$ second pause $\rightarrow$ Can you think of any more creative things you could use a pencil for? 
Once participant communicates s/he can no longer think of more responses, transition to next task.

\section{Instances Task}

3.1. Display marble on screen and hold marble to camera. Do you know what this is?

$>$ If unsure/unresponsive/inaccurate: This is a marble/marbles are round

$>$ If accurate: That's right! [reaffirm child's response].

3.2. Can you think of anything else that is round?

$>$ If unsure/unresponsive/inaccurate: [draw circle on paper] this is a circle. Just like a marble, circles are round.

$>$ If accurate: That's right! [reaffirm child's response].

3.3. In this game, I want you to tell me all of the things you can think of that are round. Try to think of all the round things you can and remember to be as creative as you can.

$>$ Positively reaffirm every response.

$>$ In the case of $a>5$ second pause $\rightarrow$ Can you think of any more things that are round?

$>$ Once participant communicates s/he can no longer think of more responses, transition to filler task. After filler task, transition to 3.4

3.4. Nice work, \{child name $\}$. Do you remember the game we were playing about things that are round? You came up with a lot of creative things that are round. You said [list participant's responses here] were all round. Can you think of any more creative things that are round?

$>$ Positively reaffirm every response.

$>$ In the case of $a>5$ second pause $\rightarrow$ Can you think of any more things that are round?

$>$ Once participant communicates s/he can no longer think of more responses, transition to next task

\section{Filler Tasks}

FT-Colors. You were great at that game, \{child name $\}$ ! Now I have a(nother) game I would like to play with you. In this game, you will see a color on the screen. I just want you to tell me what color you see

FT-Shapes. Awesome work, \{child name $\}$ ! You were really good at that game. Now I have a(nother) card game I would like to play with you. In this game, you will see a shape appear on the screen. I just want you to tell me what shape you see.

\section{Creative Drawing Task}

4.1. [Ask parent to bring out art supplies] The next game I want to play is a coloring game. I want you to draw something really creative like a magical creature or an 
imaginary friend, or maybe even something no one has ever seen or thought of before! Can you think of something really creative that you want to draw?

$>$ If unsure/unresponsive/not particularly creative, brainstorm ideas with participant until s/he identifies one

$>$ If participant articulates a creative idea $\rightarrow$ That's a great idea!

$>$ After participant indicates s/he is finished, transition to 4.2

4.2. This is a very creative drawing! [compliment features of drawing]. You know what this drawing needs? A title! Do you think you could give this drawing a creative title?

$>$ Use follow-up prompt if necessary $\rightarrow$ What is a creative name we could give this drawing?

$>$ If participant does not give drawing title use response to 4.1 "what do you think we should draw?" (Ask parent to write title on drawing). Otherwise, transition to 4.3

4.3 That is a very creative name for your drawing! [Ask parent to write title on drawing and transition to next task]

\section{Verbal Reasoning ("Guess What”) Task}

5.1. The last game I want to play is a guessing game! I'm going to give you three clues, or hints, and then I want you to guess the thing I'm talking about. Let's try a practice one. This one is a bug with no legs, you can find it in the dirt, and sometimes birds eat it. Can you get what it is!

$>$ If unsure/unresponsive/or incorrect, repeat the hints one more time. If still no (correct) answer $\rightarrow$ It's a worm! Display picture on screen, explain how it relates to the hints, and provide encouragement. You did a good job trying to figure it out. Let's try a few more!

$>$ If participant answers correctly $\rightarrow$ That's right! It's a worm! Let's try a few more

5.2. Ok, let's try another one. I'm going to give you three hints, and then I want you to try to guess what it is! It has four legs and a tail, some people like to keep it as a pet, and it has whiskers. Can you guess what it is?

$>$ If unsure/unresponsive/or incorrect, repeat the hints one more time. If still no (correct) answer $\rightarrow$ It's a cat! Display picture on screen, explain how it relates to the hints, and provide encouragement. Let's try a few more!

$\rightarrow$ If participant provides an acceptable answer $\rightarrow$ That's right! It is a \{child response\} Great guess, \{child name\} Let's try a few more

5.3. Here are your three hints. It falls from the sky, it helps plants grow, and it will get you wet if it touches you. Can you guess what it is?

$>$ If unsure/unresponsivelor incorrect, repeat the hints one more time. If still no (correct) answer $\rightarrow$ It's rain! Display picture on screen, explain how it relates to the hints, and provide encouragement. Let's try a few more!

$>$ If participant provides an acceptable answer $\rightarrow$ That's right! It is \{child response Great guess, \{child name\} Let's try two more. 
5.4. Here are your three hints: It has words in it, sometimes it has pictures, and you can find it on a shelf. Can you guess what it is?

$>$ If unsure/unresponsivelor incorrect, repeat the hints one more time. If still no (correct) answer $\rightarrow$ It's a book! Explain how it relates to the hints and then provide encouragement. Let's try a few more!

$>$ If participant provides an acceptable answer $\rightarrow$ That's right! It is \{child response\} Great guess, \{child name\} Let's try one more.

5.5. Here are your three hints: It's yellow, it grows on a tall plant that looks like a tree, and you peel it before you eat it. Can you guess what it is?

$>$ If unsure/unresponsive/or incorrect, repeat the hints one more time. If still no (correct) answer $\rightarrow$ It's a banana! Display picture on screen, explain how it relates to the hints, and provide encouragement. You were really good at that game, \{child name .

$>$ If participant provides an acceptable answer $\rightarrow$ That's right! It is \{child response $\}$ Great guess, \{child name $\}$ ! Thank you so much for playing these games with me, $\{$ child name $\}$ 


\section{Appendix C}

\section{Subjective Rating Instructions}

This appendix presents the instructions used for the subjective ratings. Instructions and criteria were adopted from Silvia et al., (2008) "Assessing Creativity with Divergent Thinking Tasks: Exploring the Reliability and Validity of New Subjective Scoring Methods." Instructions and criteria were slightly modified to align with the current study divergent thinking tasks. Raters considered these same criteria when evaluating children's drawings and titles.

\section{Rating Divergent Thinking Task Responses}

Creativity can be viewed as having three facets. Creative responses will generally be high on all three, although being low on one of them does not disqualify a response from getting a high rating. We will use a 1(not at all creative) to 5 (highly creative) scale. Please carefully read the criteria below before completing your ratings

1. Uncommon: Creative ideas are uncommon: they will occur infrequently in our sample. Any response that is given by a lot of people is common, by definition. Unique responses will tend to be creative responses, although a response given only once need not be judged as creative. For example, a random or inappropriate response would be uncommon but not creative.

2. Remote: Creative ideas are remotely linked to everyday objects and ideas. For example, creative uses for a pencil are "far from" common, everyday, normal uses for a pencil, and creative instances of things that are round are "far from" common round objects. Responses that stray from obvious ideas will tend to be creative, whereas responses close to obvious ideas will tend to be uncreative.

3. Clever: Creative ideas are often clever: they strike people as insightful, ironic, humorous, fitting, or smart. Responses that are clever will tend to be creative responses. Keep in mind that cleverness can compensate for the other facets. For example, a common use cleverly expressed could receive a high score. 


\section{Appendix D}

\section{Complete Ideational Pools for Divergent Thinking Tasks}

This appendix presents children's raw responses for alternative uses and instances tasks. Due to the size of each ideational pool, separate tables are presented for pre-incubation, short incubation, and long incubation for both DT tasks.

\section{Table D1}

Uses for a Pencil - Pre-Incubation Alphabetized Responses

\begin{tabular}{|c|c|c|c|c|c|}
\hline bake with it & draw another pencil & drop it (2) & make something & push it & use it as a measurer \\
\hline balance it on your finger & draw butts & drop them & make something cool & put it in mouth & use it as a trap \\
\hline being artistic & draw everywhere & drumming & make squares & put it on a computer & use it at school \\
\hline bite the eraser off & draw humans & erase (3) & makeup on dolls & put it on a desk & use it to flatten playdough \\
\hline bookmark & draw in the car & erase stuff & making shapes & put it on a dog & use on walls \\
\hline break it in half & draw letters & erasing (5) & measuring things & put it on a house & when I'm a big girl \\
\hline build letters & draw mini mouse & fill in erased spots & mix hot chocolate & put it on a monkey's drink & with paper (4) \\
\hline building & draw numbers & for buttons & move it in a circle & put them away & words \\
\hline color (2) & draw on a glass & handle for a sign & on paper & roll it & write (2) \\
\hline color a spider & draw on the back & have colored ones & open things with it & roll it in your hands & write a name \\
\hline color the pencil & draw on the front & hold it & outlining & rolling & write a note \\
\hline coloring $(6)$ & draw on the side & holding it on the end & paint & scribble (3) & write books (2) \\
\hline coloring a picture & draw squiggly line & homework & paint it & sharpen & write cards \\
\hline coloring an outline & drawing (26) & important stuff & paint with it & sharpen it (3) & write creative things \\
\hline coloring eyes & drawing a gift card & jab something & pick them up & sharpen them & write letters \\
\hline coloring on face & drawing a tractor & make a flag with it & play tic tac toe & sign contracts & write name of card \\
\hline cover eyes with it & drawing books & make a line & poke yourself & slide it through a tube & write notes (2) \\
\hline do not use it & drawing cats & make a pencil rainbow & pop things with it & something good & write on a wall or desk \\
\hline do projects & drawing circles (2) & make a pickle pencil & pretend it is a marker & stick a whole in a window & write on stuff \\
\hline dolls eyes & drawing comic books & make art with it & pretend it is a straw & stick it in the ground & write with your brain \\
\hline dolls head & drawing letters & make designs & pretend it's a person & tap it on a table & write with your toes \\
\hline draw (3) & drawing name & make fireworks & pretend it's a tree & tell people what it does & write words (2) \\
\hline draw a face & drawing numbers & make lines & pretend it's treasure & throw them away & writing (11) \\
\hline draw a hundred things & drawing on paper & make rectangle windows & pretend it's your pet & to learn & \\
\hline draw a Michigan State logo & drawing words in books & make shapes & pretend you're giving a shot & tracing things & \\
\hline
\end{tabular}

Note. Responses repeated by more than one child display the number of children who produced that response in parentheses. 


\section{Table D2}

Uses for a Pencil - Short Incubation Alphabetized Responses

\begin{tabular}{|c|c|c|c|c|}
\hline block a camera & draw purses & make a ghost & pretend it is a sword & shape clay \\
\hline block sound & draw silly face & make a letter "A" & pretend it is a wand & share them with friends \\
\hline break it & draw something else & make a mask & pretend it is a zoo & spaceship \\
\hline break it in a fan & draw things that are cool & make a pencil out of other & pretend to use it & stick it in a candle \\
\hline break through a light bulb & draw trees & things* ${ }^{*}$ & put a pencil in crayon & tap it on a box \\
\hline build a car & draw with it & make a square (2) & put a pencil in paper & tap it on a door \\
\hline build a house & draw your name & make a triangle & put it behind your ear & tap it on a kennel \\
\hline color a tree & draw zig zags & make it a race car & put it in a light & tap it on a phone screen \\
\hline color the floor & drawing & make letters & put it on a bunch of things & throw it away \\
\hline coloring on paper (2) & drawing trains & make numbers & put it on a phone & use it as a spoon \\
\hline curling hair & drink with it & make shapes & put it on the floor & use it in a show \\
\hline cut off eraser to make it double-ended & erase stuff & making a box & put liquid on it & use metal part to make fire \\
\hline dig holes & erase things & making squares & put your hand on it & use them as knitting sticks \\
\hline draw a toy & for my sister's room & move a cockroach & putting it in hair & with animals \\
\hline draw an animal & give it away & play & roll it around the room & with cheese \\
\hline draw birds & hold a paper towel & play with clay & roll out some dough & with either hand \\
\hline draw bushes & hold stuff & play with it & roll out some playdough & with grandpa \\
\hline draw cheeks & homework & poke holes in slime & rub it on a rock & write a story \\
\hline draw dog pop & light a fire with it & poking & save page in a book & write someone's name \\
\hline draw ears & like a comb & pretend it is a broke stick & school work & writing books \\
\hline draw helicopters & make a calendar & pretend it is a spoon & see what color it is & writing numbers \\
\hline draw on clay & make a city out of them & pretend it is a stick & set it on a box & \\
\hline
\end{tabular}

Note. Responses repeated by more than one child display the number of children who produced that response in parentheses.

Asterisks indicate responses that continue from the previous line. 


\section{Table D3}

Uses for a Pencil - Long Incubation Alphabetized Responses

\begin{tabular}{|c|c|c|}
\hline as a block & put it on a fork and roll it around & drawing \\
\hline as a fork & roll it around & drawing a map \\
\hline as a spoon & roll it back and forth with hands & to make little holes to plant plants \\
\hline bake cookies & stick it in a candle & give it back \\
\hline balance in both hands & throw it in a freezer until it's an ice cube then take it out & make a microphone \\
\hline bounce in both hands & and put it in the sun* & make a race car but we'll have to put buttons on it for the \\
\hline break it & to play games & wheels* \\
\hline throw it & to poke little holes in play dough & make a star out of them \\
\hline cannot throw it outside our window & to poke things & make an elf out of it \\
\hline color on things & to write in the space where you're battling* & on paper \\
\hline color things pencil color & tractor color & painting \\
\hline color with it & we can break it with a tree & paper \\
\hline draw Christmas trees & we could write NSG & pretend it's a guy \\
\hline draw my hand & write letters and numbers too & scribble \\
\hline draw people & write my name & smash it on your tv \\
\hline draw shapes (2) & write on the wall & smash stuff \\
\hline draw your stuffed animal & write words & snap it apart and put it on a crayon \\
\hline drawing humans & help someone get something unstuck & tap it 1 \\
\hline erase stuff with it & you can roll it & to color on the paper \\
\hline hitting stuff & a pencil could be a dog & to draw an eyeball \\
\hline hot glue it and make a building & a wand & to write my name \\
\hline juggle with fun thing & a worm & use it for a mixer \\
\hline magnets & an art project & use it to unjam stuff \\
\hline make an alligator & break it & we can draw with it \\
\hline making a drum set & building & write with it upside down \\
\hline on dresses & coloring & write your name \\
\hline on puzzles & do anything I want & writing (2) \\
\hline put an arrow on it to launch it & draw & you could roll a pencil \\
\hline paint it blue and black and gray & draw notes & you could use it in battle to throw it when it's sharp to \\
\hline pin stuff & draw pictures & poke them* \\
\hline poke someone on accident & draw some draw with it & \\
\hline put it in the pencil sharpener & thing important & \\
\hline
\end{tabular}

\section{Note. Responses repeated by more than one child display the number of children who produced that response in parentheses.}

Asterisks indicate responses that continue from the previous line. 


\section{Table D4}

Things that are Round - Pre-Incubation Alphabetized Responses

\begin{tabular}{|c|c|c|c|c|c|}
\hline apples (3) & button & dot of glue & jar & paper & sticks \\
\hline arms held in a circle & buttons & dots & jars (2) & pen holder & strings like yarn \\
\hline around & cakes & drew circle & knobs (3) & pencil tips & stuffed animal \\
\hline baby bottles & cannon balls & DVD players & lamp shades & pencils & $\operatorname{sun}(5)$ \\
\hline baby toys & cap for something & ears (2) & lamps (2) & pictures & sunglasses (2) \\
\hline bagels & cardboard rocket & earth (2) & lemons & pigs & teeth \\
\hline bags & carpets & end of a marker & letter "o" (6) & planets (3) & the top of nails \\
\hline ball (5) & $\operatorname{cars}(2)$ & exercise balls & lid for a marker & plates & tip of an arrow \\
\hline balloons & chapstick & eye & lid on lotion & playdough & tire on a car \\
\hline balls (22) & cheerios (3) & eyes (10) & lids (4) & ponytail bands & tires \\
\hline baseball & circle (2) & fans & light bulb & pools & top of a can \\
\hline basketball (2) & circle frames & fat dogs & light fixture & popcorn balls & top of a crayon \\
\hline basketballs & circle stickers & flowers & lightbulbs (2) & rings & top of a lightbulb \\
\hline beach ball & circles $(10)$ & football & lights (5) & rock & top of lotion \\
\hline beach balls & clock & full moon & lights outside & rocks & torch light \\
\hline beads (3) & clocks & glass & made lips in a kissy face & rose on a bow & tracks in the snow \\
\hline bellies & coffee pots & glasses lens & little balls & round things on wires & tractors \\
\hline big circle & colors & grapes & made circle with hands & scissors handles & trees \\
\hline bigger balls & compass & gumballs & markers (5) & sharks & tubes \\
\hline birds' nests & cookies (2) & hair (2) & mirrors & shoes & wagon tire \\
\hline blankets & corks & hair ties & moon & signs & water bottle \\
\hline blue ball & corners & handle on a drawer & mouth & snow globes & water fountains \\
\hline blue berries & cupcakes (2) & handles & necks & snowballs & wheel \\
\hline bottles (2) & cups & hats (2) & noses & soccer balls (3) & wheels (6) \\
\hline bottom of a marker & deer neck & head (5) & nostrils (2) & sound machine & wheels on trucks \\
\hline bouncy ball (2) & did somersault & heads (7) & open mouth & spun in circle & wine glass \\
\hline bouncy balls (2) & dog's heads & holes (2) & oranges (3) & squishy ball & you are round \\
\hline bowls (2) & donuts (2) & huge circle & ornaments & statues & zebra's nose \\
\hline bracelets & doorknob & human neck & pacifiers & steering wheels & zero \\
\hline bushes & doorknobs (2) & inside bottle & palm of your hand & stickers on bottles & \\
\hline
\end{tabular}

Note. Responses repeated by more than one child display the number of children who produced that response in parentheses.

Italicized text indicates behavioral responses. 


\section{Table 5D}

Things that are Round - Short-Incubation Alphabetized Responses

\begin{tabular}{|c|c|c|c|c|c|}
\hline a bunny's tail & car wheels & eyelids & leaf & pillows & tooth \\
\hline a fist & CDs & eyes (2) & leaves & planets & tootie fruitie \\
\hline a hole on a coffee cup & chairs & faces & lens on a camera & plant holders & top of a nail \\
\hline a hole on a straw & chocolate & fans & letter "o" & plate & top of a stool \\
\hline a water cup & circle buttons & faucets & lid & plates & top of a top \\
\hline an eye & circle eggs & fingernails & light button & Pluto & tops of chalk (2) \\
\hline ant traps & circles $(4)$ & flashlights & lights & pockets & toy trucks \\
\hline apples & circles inside of a square & floaties & lint & Polka dot & toys \\
\hline baby toys & circles made of fuse beads & flowers (2) & $\log o s$ & poles & traffic lights \\
\hline backpacks & circles on a ceiling & fruits & markers & pools & truffles \\
\hline ball & circles on an "8" & fuse beads & Mars & poop & tummies \\
\hline balls & clay & glasses & mats & pop socket & tuna can \\
\hline basketball hoops & clocks (2) & globes & middle of flower & popcorn & Uranus \\
\hline beads & coconuts & glue stick (2) & monkey eyes & power wheel & vegetables \\
\hline blanket rolled into a ball & coffee & gumballs & monkeys & pumpkins & wheels (4) \\
\hline bolts & coffee mugs & Halloween skateboards & moon (2) & puppy dogs & windows \\
\hline bones & cords & handrails & mushrooms & quarter & yarmulkes \\
\hline books & crews & hands & nails & rafts & yoyos \\
\hline bottom of jar & cup holders & hats & napkins & rain & zero (2) \\
\hline bottle of apple juice & cups $(5)$ & heads (3) & octopus & rectangle & \\
\hline bottom of a trashcan & disco balls & hearts & oranges & rectangles & \\
\hline bowl & dogs & hedgehogs & paddleboard ball & rubber bands & \\
\hline bowling balls & donuts & helicopter toy & paint balls & Saturn & \\
\hline bowls (3) & doorknobs (2) & honey nut cheerios & paintings & scissor holes & \\
\hline bracelets & dot & inside of a bowl & paper & sinks & \\
\hline bubble tops & dots & jack-o-lantern & paper towels & spears & \\
\hline bubbles & earrings & jars & paws & steering wheels (2) & \\
\hline buttons & earth & Jupiter & pencil & $\operatorname{sun}(3)$ & \\
\hline candles (2) & eraser & lamp & pencils & tires & \\
\hline caps & erasers & lamps & pets & toes & \\
\hline
\end{tabular}

\footnotetext{
Note. Responses repeated by more than one child display the number of children who produced that response in parentheses.
} 


\section{Table 6D}

Things that are Round - Long-Incubation Alphabetized Responses

\begin{tabular}{|c|c|c|c|c|c|}
\hline Alexa speaker & circles on LEGOS & grandma & nails & presents & tail \\
\hline apple (3) & circular part of a & grapes & nest & pretty dragon fruit & tape \\
\hline apples & transformer* & gumball & nose (2) & pumpkins (2) & teddies \\
\hline baby toy & climbing block & hat & $\operatorname{noses}(2)$ & railings & teapot \\
\hline ball (2) & clouds & head (4) & orange (2) & red stop sign & the back of my heel is \\
\hline balloon & coin & heads & oranges & rice & round \\
\hline balls (4) & cookie & helmets & ornament & rolled up blankets & the circle trees \\
\hline basketball & $\operatorname{cup}(3)$ & hole & ornaments & roller & the ends of your sleeves \\
\hline bath bomb & cupholder & holes & our ankles & Santa's nose & the inside of our ears \\
\hline bead & cups & jingle bell & our ears & sirens & the little dot on the tv that \\
\hline bean bag & Daisy's ball & jingle bells & our face & sled & lets us know if it's on or* \\
\hline beet & deer bodies & knob on headboard & our neck & smiley face & off* \\
\hline berries & $\operatorname{dot}$ & LEGO ball that opens & our nose holes & snow globe & the middle of a flower \\
\hline beyblade & dragon tears & LEGO head & our round arms & snow man & the other fruit like a lime \\
\hline bologna goes around in & earmuffs & lens of glasses & our toes & soccer ball & that's yellow* \\
\hline circles* & earring & letter "o" (2) & ovals & soccer balls & the thing on the remote \\
\hline bowl (5) & Easter eggs & $\operatorname{lid}(3)$ & pacifiers & some leaves on trees & the world (2) \\
\hline Bread & egg & lids & paint brush & space that holds a ball on a & tomatoes \\
\hline bunny tail & eggs (2) & light & paper plates & catapult & top of a LEGO lamppost \\
\hline bunny tails & elf's head & lightbulbs (2) & part of a heart & speakers & top of road cones \\
\hline button & eye & lights (3) & pear & spinner toy & trashcan $(2)$ \\
\hline buttons & eyeball (2) & lime & peas & spoon & trees \\
\hline butts & eyeballs & marble & pillows & spoon's head & vase \\
\hline cakes & eyes (2) & marker & pizza & spots $(2)$ & water bottle \\
\hline cannonball & fingers & markers & plain ball & stoplights & watermelon \\
\hline cap & fish & masking tape & planet & stop sign & wheel (2) \\
\hline carrots & flashlights & Mickey Mouse ears & plate & stove knob & wheels (6) \\
\hline chicken nugget & flower (2) & Mickey Mouse nose & plates & stroller wheels & wood \\
\hline Christmas lights & flowers & middle of a flower & playmobil toy & square & yellow marble \\
\hline cinnamon roll & freckle & mommy's eye & polka dots & $\operatorname{sun}(2)$ & your eyes \\
\hline circle (2) & frosty the snowman & moon (2) & pom poms & table & zero $(2)$ \\
\hline circles (3) & glasses & my owl's head & pot & table downstairs & \\
\hline
\end{tabular}

Note. Responses repeated by more than one child display the number of children who produced that response in parentheses. Asterisks indicate responses that continue from the previous line. 


\section{Appendix E}

\section{Linear Regression Assumptions Tests}

This appendix presents visual and statistical evidence for the normality (i.e., Shapiro-Wilk tests and Normal P-P Plots of standardized residuals), and homoscedasticity of residuals (i.e., scatterplots) and, in the case of multiple regression, the absence of multicollinearity issues (VIF).

\section{Model 1: Age and Fluency as Predictors of AUT Short PO}
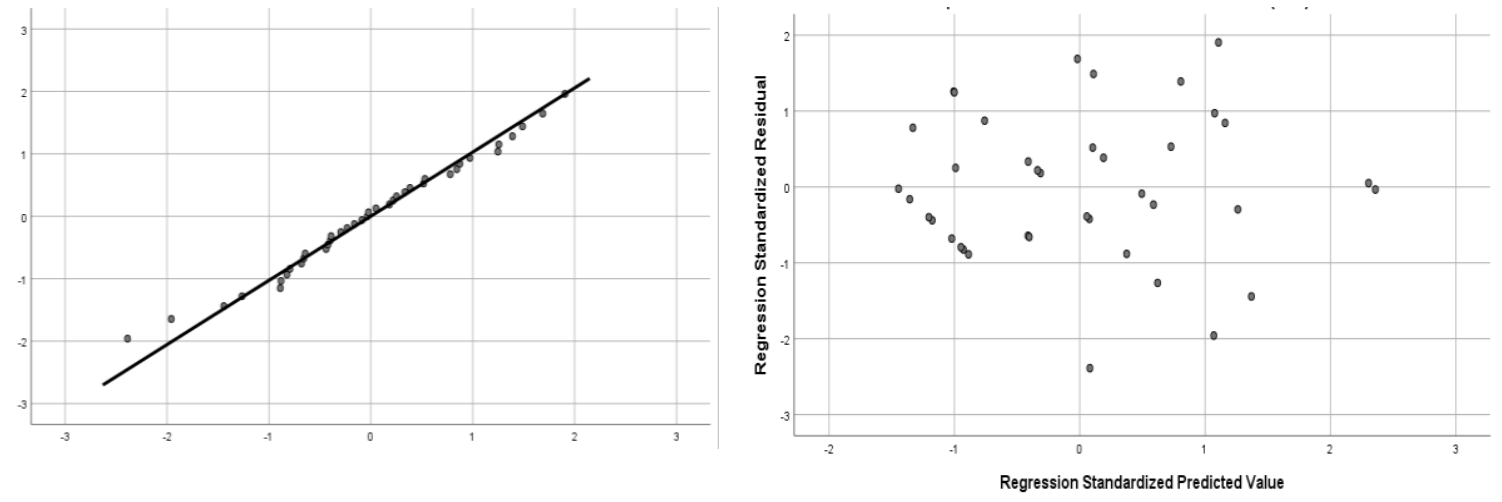

Shapiro-Wilk Test Result: $\mathrm{W}(39)=.987, p=.92 \mid \mathrm{VIF}=1.13$

\section{Model 2: Age as a Predictor of IT Short PO}
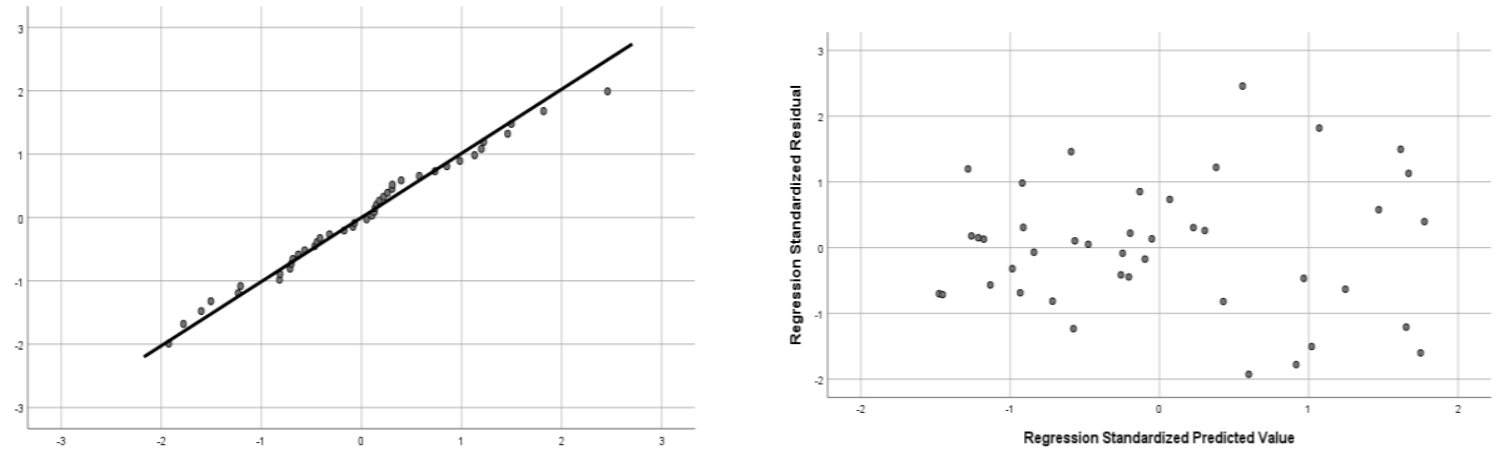

Shapiro-Wilk Test Result: W(42) $=.987, p=.90$. 
Model 3: Age and Fluency as Predictors of IT Long PO
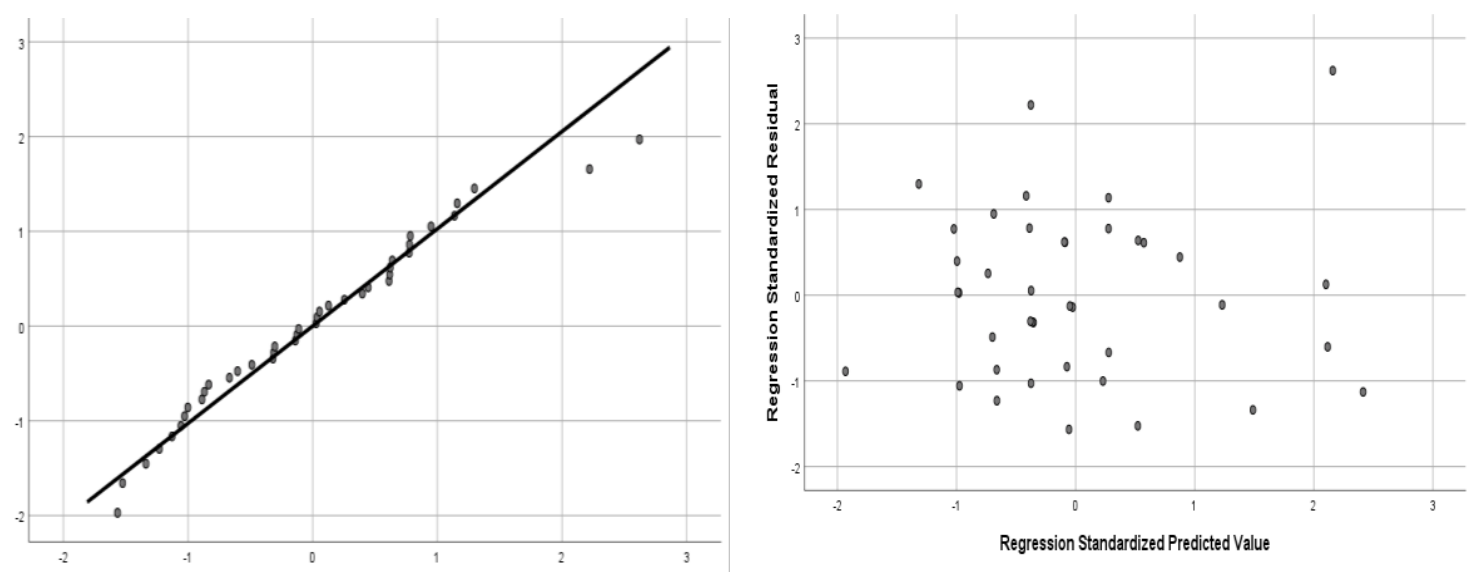

Shapiro-Wilk Test Result: $\mathrm{W}(40)=.965, p=.256 \mid \mathrm{VIF}=1.20$ 
VITA

James A. Larsen earned his B.S. in Human Development and Family Studies from the University of Missouri in 2014. Under the supervision of Dr. Louis Manfra, James proceeded to earn his M.S. in Human Development and Family Science in 2018, and his Ph.D. in Human Environmental Sciences, with a collateral in Quantitative Methods, in 2021. 